\title{
On the automorphism group of generalized Baumslag-Solitar groups
}

\author{
GiLBERT LEVITT
}

\begin{abstract}
A generalized Baumslag-Solitar group (GBS group) is a finitely generated group $G$ which acts on a tree with all edge and vertex stabilizers infinite cyclic. We show that $\operatorname{Out}(G)$ either contains non-abelian free groups or is virtually nilpotent of class $\leq 2$. It has torsion only at finitely many primes.

One may decide algorithmically whether $\operatorname{Out}(G)$ is virtually nilpotent or not. If it is, one may decide whether it is virtually abelian, or finitely generated. The isomorphism problem is solvable among GBS groups with $\operatorname{Out}(G)$ virtually nilpotent.

If $G$ is unimodular (virtually $F_{n} \times \mathbb{Z}$ ), then $\operatorname{Out}(G)$ is commensurable with a semidirect product $\mathbb{Z}^{k} \rtimes \operatorname{Out}(H)$ with $H$ virtually free.
\end{abstract}

20F65; 20E08, 20F28

\section{Introduction and statement of results}

The groups $B S(m, n)=\left\langle a, t \mid t a^{m} t^{-1}=a^{n}\right\rangle$ were introduced by Baumslag-Solitar [2] as very simple examples of non-Hopfian groups (a group $G$ is non-Hopfian if there exists a non-injective epimorphism from $G$ to itself). It is now known that $B S(m, n)$ is Hopfian if and only if $m= \pm 1$, or $n= \pm 1$, or $m, n$ have the same set of prime divisors (Collins-Levin [7]). In particular, $B S(2,4)$ is Hopfian while $B S(2,3)$ is not.

Though it has exotic epimorphisms, $B S(2,3)$ has very few automorphisms: its automorphism group is generated by inner automorphisms and the obvious involution sending $a$ to $a^{-1}$ (Collins [6] and Gilbert et al [14]). On the other hand, BS $(2,4)$ has an incredible number of automorphisms, as its automorphism group is not finitely generated [7].

The reason behind this drastic difference is that, because 2 divides 4 but not 3, the presentation of $B S(2,4)$ is much more flexible than that of $B S(2,3)$. By this we mean, in particular, that $B S(2,4)$ admits the infinite sequence of presentations $\left(1_{p}\right)$

$$
B S(2,4)=\left\langle a, b, t \mid t b t^{-1}=b^{2}, b^{2^{p}}=a^{2}\right\rangle
$$


obtained from the standard one by introducing a new generator $b=t^{-p} a^{2} t^{p}$. It is clear already from $\left(1_{p}\right)$ that $G=B S(2,4)$ has many automorphisms, as fixing $b, t$ and conjugating $a$ by $b$ defines an element of order $2^{p}$ in $\operatorname{Out}(G)$.

The presentations $\left(1_{p}\right)$ express $B S(2,4)$ as a generalized Baumslag-Solitar group, or $G B S$ group, or graph of $\mathbb{Z}$ 's, namely as the fundamental group of a finite graph of groups $\Gamma$ with all edge and vertex groups infinite cyclic. This is visualized as a labelled graph, with the absolute value of the labels indicating the index of edge groups in vertex groups (see Figure 1).
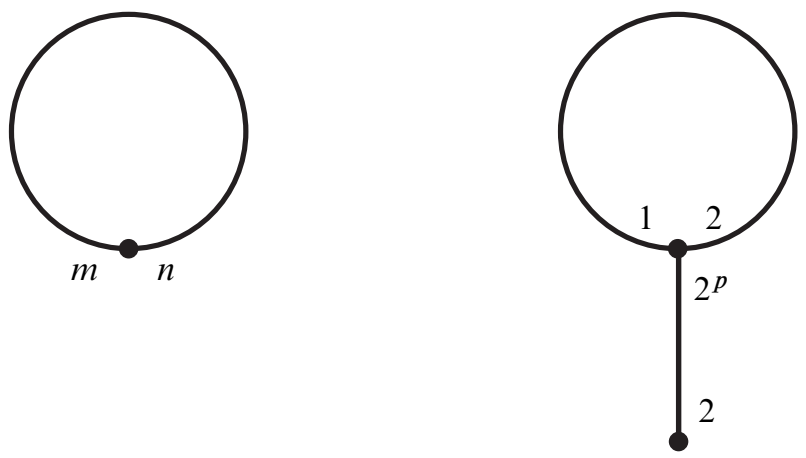

Figure 1: The labelled graphs associated to the standard presentation of $B S(m, n)$, and to $\left(1_{p}\right)$.

In this paper, we study automorphisms of GBS groups. See Forester [12; 13], Kropholler [18], Levitt [21] and Whyte [34] for various algebraic and geometric properties of these groups. As pointed out in [12], they are especially interesting in connection with JSJ theory.

Before giving general results, let us review certain classes of GBS groups for which more specific statements may be obtained. They are defined either by "local" conditions on the labelled graph, or by "global" algebraic conditions on the group. In the rest of this introduction, we always assume that $G$ is not one of the elementary GBS groups: $\mathbb{Z}, \mathbb{Z}^{2}$, and the Klein bottle group.

\section{Algebraically rigid groups}

As evidenced by the example of $B S(2,4)$, the main difficulty with GBS groups is that they may be represented by many different labelled graphs $\Gamma$. Sometimes, though, $\Gamma$ is essentially unique. By (Gilbert [14]) and (Pettet [30]), this algebraic rigidity holds in particular when there is no divisibility relation in $\Gamma$ : if $p, q$ are labels near the 
same vertex, then $p$ does not divide $q$ (see Section 2 for a precise definition and a characterization of algebraic rigidity).

Given $\Gamma$, let $T$ be the associated Bass-Serre tree, which we call a GBS tree. Let $\operatorname{Out}^{T}(G) \subset \operatorname{Out}(G)$ be the subgroup leaving $T$ invariant. Most elements of $\operatorname{Out}^{T}(G)$ may be viewed as "twists" (see Section 3). Algebraic rigidity implies $\operatorname{Out}^{T}(G)=$ $\operatorname{Out}(G)$, but in general $\operatorname{Out}^{T}(G)$ is smaller.

Theorem 1.1 Let $G$ be a GBS group, represented by a labelled graph $\Gamma$, and let $T$ be the Bass-Serre tree. Define $k$ as the first Betti number $b$ of $\Gamma$ if $G$ has a non-trivial center, as $b-1$ if the center is trivial.

(1) The torsion-free rank of the abelianization of $G$ is $k+1$.

(2) The group $\operatorname{Out}^{T}(G)$ is virtually $\mathbb{Z}^{k}$.

(3) Up to commensurability within $\operatorname{Out}(G)$, the subgroup $\operatorname{Out}^{T}(G)$ does not depend on $\Gamma$.

Conversely, any subgroup of $\operatorname{Out}(G)$ commensurable with a subgroup of $\operatorname{Out}^{T}(G)$ is contained in $\mathrm{Out}^{T^{\prime}}(G)$ for some GBS tree $T^{\prime}$ (Clay [5]).

For $G=B S(m, n)$, one has $k=0$ if $m \neq n$, and $k=1$ if $m=n$. For $G=B S(2,4)$ with the presentation $\left(1_{p}\right)$, the group $\operatorname{Out}^{T}(G)$ has order $2^{p+1}$.

Corollary 1.2 If $G$ is algebraically rigid, then $\operatorname{Out}(G)$ is virtually $\mathbb{Z}^{k}$.

The converse is also true if $G$ is not solvable (see Theorem 8.5).

\section{Unimodular groups}

A GBS group $G$ is unimodular if $x y^{p} x^{-1}=y^{q}$ with $y \neq 1$ implies $|p|=|q|$, or equivalently if $G$ is virtually $F_{n} \times \mathbb{Z}$ (with $F_{n}$ a free group of rank $n$ ). The group $G$ then has a normal infinite cyclic subgroup with virtually free quotient, and we show the following theorem.

Theorem 1.3 If $G$ is unimodular, there is a split exact sequence

$$
\{1\} \rightarrow \mathbb{Z}^{k} \rightarrow \text { Out }_{0}(G) \rightarrow \text { Out }_{0}(H) \rightarrow\{1\},
$$

where $k$ is as above, $H$ is virtually free, and $\mathrm{Out}_{0}$ has finite index in Out.

Since $\operatorname{Out}(H)$ is VF (Krstić-Vogtmann [20]), we get the following Corollary.

Corollary 1.4 $\operatorname{Out}(G)$ is virtually torsion-free and VF (it has a finite index subgroup admitting a finite classifying space). 


\section{Groups with no non-trivial integral modulus}

Now consider groups $G$ which do not contain a solvable Baumslag-Solitar group $B S(1, n)$ with $n \geq 2$ (there is an equivalent characterization in terms of the modular homomorphism $\Delta: G \rightarrow \mathbb{Q}^{*}$, see Section 2).

Given any GBS group $G$, the group $\operatorname{Out}(G)$ acts on the space $P \mathcal{D}$ of all GBS trees (see Section 5), with stabilizers virtually $\mathbb{Z}^{k}$ by Theorem 1.1. Clay [4] proved that the space $P \mathcal{D}$ is contractible (see also Guirardel-Levitt [16]) and Forester [13] proved that the quotient is a finite complex if $G$ does not contain $B S(1, n)$ with $n \geq 2$. This gives the following Theorem.

Theorem 1.5 If $G$ does not contain $B S(1, n)$ for $n \geq 2$, then $\operatorname{Out}(G)$ is $F_{\infty}$ (in particular, it is finitely presented). If furthermore $\operatorname{Out}(G)$ is virtually torsion-free, then it is VF.

\section{Arbitrary groups}

Now let $G$ be any GBS group.

Theorem 1.6 Either $\operatorname{Out}(G)$ contains a nonabelian free group, or it is virtually nilpotent of class $\leq 2$.

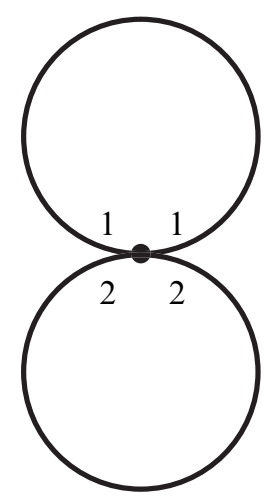

Figure 2: $\operatorname{Out}(G)$ is virtually the integral Heisenberg group $H_{3}$.

A group is nilpotent of class $\leq 2$ if and only if every commutator is central. As an example, let $G=\left\langle a, s, t \mid s a=a s, t a^{2}=a^{2} t\right\rangle$ (see Figure 2). Then $\operatorname{Out}(G)$ is virtually the integral Heisenberg group $H_{3}$, with

$$
\left(\begin{array}{lll}
1 & i & j \\
0 & 1 & k \\
0 & 0 & 1
\end{array}\right)
$$


mapping $(a, s, t)$ to $\left(a, s a^{k}, t s^{i} a^{j}\right)$.

Which possibility of Theorem 1.6 occurs may be explicitly decided from the divisibility relations in any labelled graph $\Gamma$ representing $G$. We have seen that $\operatorname{Out}(G)$ is virtually abelian if there is none. A key observation is that certain divisibility relations force the existence of $F_{2}$ inside $\operatorname{Out}(G)$.

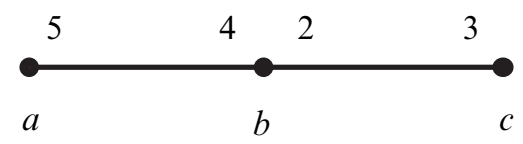

Figure 3: $\operatorname{Out}(G)$ contains $F_{2}$.

As a basic example, consider $G=\left\langle a, b, c \mid a^{5}=b^{4}, b^{2}=c^{3}\right\rangle$ (see Figure 3). It is the amalgam of $G_{1}=\langle a\rangle$ with $G_{2}=\langle b, c\rangle$ over $C=\left\langle b^{4}\right\rangle$. The divisibility relation $2 \mid 4$ at the middle vertex implies that $C$ is central in $G_{2}$. For any $g \in G_{2}$, we may therefore define an automorphism $\varphi_{g}$ of $G$ as being the identity on $G_{1}$ and conjugation by $g$ on $G_{2}$. It is easy to show that the subgroup of $\operatorname{Out}(G)$ generated by the $\varphi_{g}$ 's is isomorphic to $\left\langle b, c \mid b^{2}=c^{3}=1\right\rangle$, hence contains $F_{2}$.

To prove Theorem 1.6, we assume that $\operatorname{Out}(G)$ does not contain $F_{2}$ and we describe which divisibility relations may occur (Section 6). In Section 7, we show that, though the GBS tree $T$ may not be $\operatorname{Out}(G)$-invariant, some (non GBS) tree $S$ obtained from $T$ by collapsing certain edges is. We then prove that $\operatorname{Out}^{S}(G)$ is virtually nilpotent.

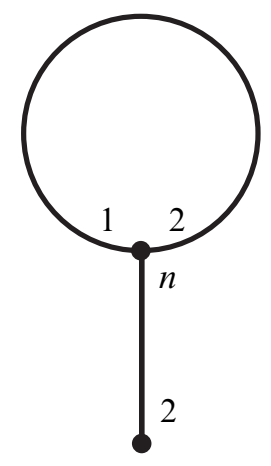

Figure 4: $\operatorname{Out}(G)$ is virtually an infinitely generated abelian group if $n$ is not a power of 2 .

For instance, let $G=\left\langle a, b, t \mid t b t^{-1}=b^{2}, b^{n}=a^{2}\right\rangle$ with $n$ not a power of 2 (see Figure 4). In this case, $S$ is obtained from $T$ by collapsing edges projecting onto the loop of $\Gamma$. The group $\operatorname{Out}(G)$ is virtually abelian (but not finitely generated).

A special case of Theorem 1.6 is the following Theorem. 
Theorem 1.7 If no label of $\Gamma$ equals 1 , then $\operatorname{Out}(G)$ contains $F_{2}$ or is a finitely generated virtually abelian group.

As a corollary of our analysis, we show the following Theorem.

Theorem 1.8 The isomorphism problem is solvable for GBS groups $G$ such that $\operatorname{Out}(G)$ does not contain $F_{2}$.

The isomorphism problem for GBS groups is to decide whether two labelled graphs define isomorphic groups. It is solvable for groups with no non-trivial integral modulus [13] and 2-generated groups [21], but open in general.

We also show the following.

Theorem 1.9 The set of prime numbers $p$ such that $\operatorname{Out}(G)$ contains non-trivial $p$-torsion is finite.

The paper is organized as follows. In Section 2, we review basic properties of GBS groups, such as algebraic rigidity and the modular homomorphism $\Delta$. We extend to GBS groups a result of $\mathrm{Fel}^{\prime}$ shtyn-Goncalves [10] about twisted conjugacy classes. In Section 3, we study $\operatorname{Out}^{T}(G)$, proving Theorem 1.1 and Theorem 1.9. Section 4 is devoted to unimodular groups, Section 5 to the action of $\operatorname{Out}(G)$ on $P \mathcal{D}$. Theorem 1.6 is proved in Section 6 and Section 7. In Section 8 we discuss several special cases and the isomorphism problem. Section 9 contains open questions.

\section{Acknowledgements}

I am grateful to M Forester and M Clay for useful suggestions and help with the section on open questions and to P Papasoglu for pointing out that $\operatorname{Out}(B S(2,4))$ is not finitely generated.

\section{Basic facts about GBS groups}

\section{Labelled graphs}

A GBS group $G$ is the fundamental group of a finite graph of groups $\Gamma$ whose vertex and edge groups are all infinite cyclic. It is torsion-free. Topologically, $G$ is the fundamental group of a 2-complex consisting of annuli (corresponding to edges of $\Gamma$ ) glued to circles (corresponding to vertices). 
We denote by $b$ the first Betti number of the graph $\Gamma$. Note the distinction between $G=\pi_{1}(\Gamma)$ and the topological fundamental group $\pi_{1}^{\text {top }}(\Gamma) \simeq F_{b}$.

If we choose generators for edge and vertex groups, the inclusion maps are multiplications by non-zero integers. An oriented edge $e$ thus has a label $\lambda_{e} \in \mathbb{Z} \backslash\{0\}$, describing the inclusion of the edge group $G_{e}$ into the vertex group $G_{o(e)}$ at the origin of $e$. As in [13], we visualize the graph of groups as a labelled graph $\Gamma$, with the label $\lambda_{e}$ pictured near the origin $o(e)$.

A pair $\varepsilon=(e, \bar{e})$ of opposite edges is a non-oriented edge. It carries two labels, one near either endpoint $o(e), o(\bar{e})$, and we say that $\varepsilon$ (or $e$ ) is a $\left(\lambda_{e}, \lambda_{\bar{e}}\right)-e d g e$. An edge is a loop if its endpoints are equal, a segment if they are distinct.

The group $G$ associated to a labelled graph $\Gamma$ may be presented as follows. Choose a maximal subtree $\Gamma_{0} \subset \Gamma$. There is one generator $x_{v}$ for each vertex $v$, and one generator $t_{\varepsilon}$ for each non-oriented edge $\varepsilon$ not in $\Gamma_{0}$. Each non-oriented edge $\varepsilon=(e, \bar{e})$ of $\Gamma$ contributes one relation. If $\varepsilon$ is contained in $\Gamma_{0}$, the relation is $\left(x_{o(e)}\right)^{\lambda(e)}=\left(x_{o(\bar{e})}\right)^{\lambda(\bar{e})}$. If $\varepsilon$ is not in $\Gamma_{0}$, the relation is $t_{e}\left(x_{o(e)}\right)^{\lambda(e)} t_{e}^{-1}=\left(x_{o(\bar{e})}\right)^{\lambda(\bar{e})}$.

Replacing the chosen generator of a vertex group $G_{v}$ by its inverse changes the sign of all labels near $v$. Replacing an edge group generator changes the sign of both labels carried by the edge. These changes are admissible sign changes. Labelled graphs will always be considered up to admissible sign changes.

When we focus on a particular edge, we always use admissible sign changes to make it a $(p, q)$-segment with $p, q>0$, or a $(p, q)$-loop with $1 \leq p \leq|q|$.

A $(1, q)$-loop is an ascending loop. It is a strict ascending loop if $|q|>1$; note that $G$ then contains a solvable Baumslag-Solitar group $B S(1, q)$. A $(p, q)$-loop with $p \mid q$ is a pseudo-ascending loop.

\section{GBS trees}

Let $G$ be the fundamental group of a labelled graph $\Gamma$. The associated Bass-Serre tree is a locally finite $G$-tree $T$ with all edge and vertex stabilizers infinite cyclic. Such $G$-trees will be called GBS trees. Two trees are considered to be the same if there is a $G$-equivariant isomorphism between them.

We always assume that the action is minimal: there is no proper $G$-invariant subtree. In terms of $\Gamma$, this is equivalent to saying that the label near every terminal vertex is bigger than 1. We also assume that actions are without inversions.

Given a GBS tree $T$, one obtains a labelled graph $\Gamma=T / G$, with the labelling well-defined up to admissible sign changes [13, Remark 2.3]. This graph of groups is 
marked: there is an isomorphism from its fundamental group to $G$, well-defined up to composition with an inner automorphism. The valence of a vertex $v \in T$ is the sum of the absolute values of the labels near its image in $\Gamma$.

GBS trees $T$ and marked labelled graphs $\Gamma$ are thus equivalent concepts. We will work with both. We usually use the same letter $v$ (resp. $e$ ) for a vertex (resp. edge) of $T$ and its image in $\Gamma$. When we need to distinguish, we write $\bar{v}$ for the image of $v$ in $\Gamma$. We denote vertex stabilizers (vertex groups) by $G_{v}$, edge stabilizers (edge groups) by $G_{e}$.

\section{Collapses and algebraic rigidity}

Collapsing an edge $e$ of $\Gamma$ (or equivalently a $G$-orbit of edges of $T$ ) yields a new tree $S$, which usually is not a GBS tree. It is a GBS tree if and only if $e$ is a segment and at least one of the labels $\lambda_{e}, \lambda_{\bar{e}}$ equals 1 . Such an edge will be called a collapsible edge.

In the proof of Theorem 1.6, we will collapse $(2,2)$-edges and $(1, q)$-loops; these are not collapsible edges. We usually denote by $\Theta$ the collapsed graph of groups, by $\pi: T \rightarrow S$ the collapse map. The image of a vertex $v \in T$ is denoted by $\pi(v)$, or sometimes just $v$. The stabilizer of $\pi(v)$ in $S$ contains the cyclic group $G_{v}$, we call it $H_{v}$ (it will often be a solvable Baumslag-Solitar group). We use the same letter for a non-collapsed edge of $T$ and its image in $S$. It has the same stabilizer in both trees.

Collapsing a collapsible edge is called an elementary collapse. The reverse move is an elementary expansion. Labels near $o(e)$ get multiplied by $\lambda_{\bar{e}}$ when we collapse an edge $e$ with $\lambda_{e}=1$ (see Figure 5).

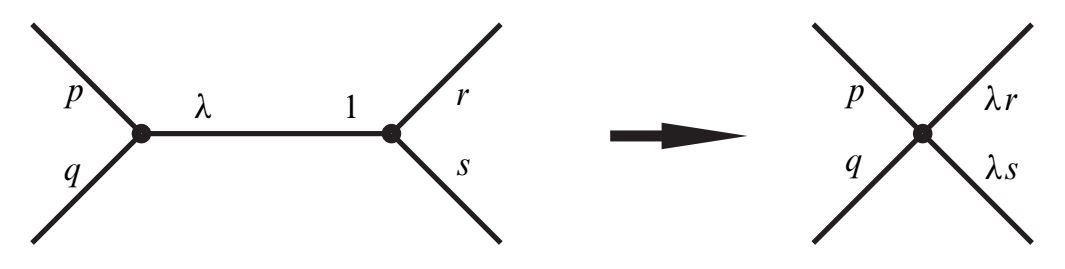

Figure 5: Elementary collapse.

The graph $\Gamma$, or the tree $T$, is reduced (in the sense of Forester [11]) if there is no collapsible edge. In terms of trees, $T$ is reduced if and only if any edge $e=v w$ satisfying $G_{e}=G_{v}$ has its endpoints in the same $G$-orbit. Any tree may be reduced by applying a finite sequence of elementary collapses (the reduction is not always unique).

A reduced GBS tree $T$ is rigid if it is the only reduced GBS tree (up to equivariant isomorphism). Building on work from Forester [11], Gilbert et al [14], Guirardel [15] 


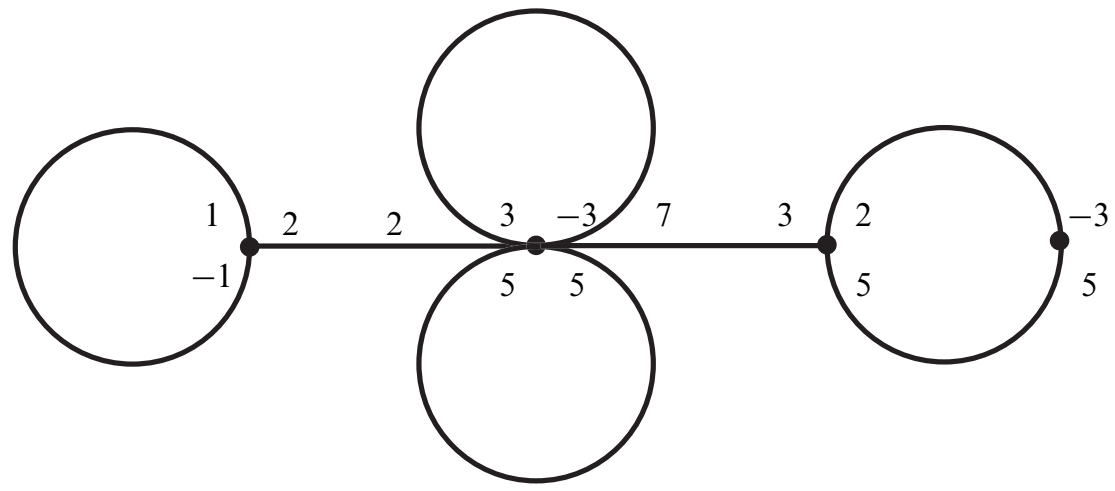

Figure 6: A labelled graph representing an algebraically rigid group.

and Pettet [30], it is shown in Levitt [23] that, if $G$ is not solvable, $T$ is rigid if and only if $\Gamma$ satisfies the following condition (see Figure 6): if $e, f$ are distinct oriented edges of $\Gamma$ with the same origin $v$, and the label of $f$ near $v$ divides that of $e$, then either $e=\bar{f}$ is a $(p, \pm p)$-loop with $p \geq 2$, or $v$ has valence 3 and bounds a $(1, \pm 1)-$ loop.

In particular, $T$ is rigid whenever there is no divisibility relation in $\Gamma$ (recall that a divisibility relation is a relation $p \mid q$ between two labels at the same vertex).

When there is a rigid GBS tree, we say that $G$ is algebraically rigid. In this case, there is only one reduced marked labelled graph representing $G$. See Mosher-Sageev-Whyte [28] and Whyte [34] for quasi-isometric rigidity of GBS groups.

\section{Non-elementary groups}

We say that $G$ is elementary if $T$ may be chosen to be a point or a line. As vertices of $T$ then have valence at most 2 , there are only four possibilities for $\Gamma$ : a point, a $(1,1)$-loop, a $(1,-1)$-loop, a $(2,2)$-segment. The corresponding groups are $\mathbb{Z}, \mathbb{Z}^{2}$, and the Klein bottle group $\left\langle x, t \mid t x t^{-1}=x^{-1}\right\rangle=\left\langle a, b \mid a^{2}=b^{2}\right\rangle$, with $\operatorname{Out}(G)$ equal to $\mathbb{Z} / 2 \mathbb{Z}, G L(2, \mathbb{Z})$, and $\mathbb{Z} / 2 \mathbb{Z} \times \mathbb{Z} / 2 \mathbb{Z}$ respectively.

Though non-elementary, the solvable groups $B S(1, n)$ are special. For $|n|>1$, the group $\operatorname{Out}(B S(1, n))$ is virtually $\mathbb{Z}^{r-1}$, where $r$ is the number of prime divisors of $n$ [6]. More generally, see $[6 ; 7 ; 14]$ for a presentation of $\operatorname{Out}(B S(m, n))$.

From now on, we consider only non-elementary groups. Here are a few simple properties (compare Forester [12]).

From the action of $G$ on $T$, it is easy to see that a non-elementary GBS group either is a solvable Baumslag-Solitar group $B S(1, n)$ (if it fixes an end of $T$ ), or contains 
non-abelian free groups (if the action on $T$ is irreducible). In particular, $G$ always has exponential growth. A finitely generated subgroup of $G$ is free (if it acts freely on $T$ ) or is a GBS group. A non-elementary GBS group is one-ended, coherent and has cohomological dimension 2 (Forester [12], Kropholler [18]).

Any GBS group maps onto $\mathbb{Z}$ (the presentation of $G$ given earlier has more generators than relators). GBS groups are therefore locally indicable, hence orderable (see Rhemtulla-Rolfsen [33]). Using the fact that $\left\langle a, b \mid a^{p}=b^{q}\right\rangle$ is bi-orderable only if $|p|$ or $|q|$ equals 1 , one shows that the only bi-orderable GBS groups are $F_{n} \times \mathbb{Z}$ and $B S(1, n)$ for $n \geq 1$.

\section{Elliptic elements}

Two subgroups $H, K$ of $G$ are commensurable if $H \cap K$ has finite index in both $H$ and $K$. Two elements $g, h$ are commensurable if $\langle g\rangle$ and $\langle h\rangle$ are commensurable, equivalently if there is a relation $g^{p}=h^{q}$ with $p, q$ non-zero integers. The commensurator of $g$ is the subgroup $\operatorname{Comm}(g)$ consisting of all $x \in G$ such that $x g x^{-1}$ is commensurable to $g$.

Given any $G$-tree $T$, an element $g \in G$, or a subgroup $H$, is elliptic if it fixes a point. If $g$ is not elliptic, it is hyperbolic: there is an invariant axis, on which $g$ acts as a translation by some positive integer $\ell(g)$. Conjugate or commensurable elements have the same type (elliptic or hyperbolic). A relation $g a^{p} g^{-1}=a^{q}$ with $|p| \neq|q|$ implies that $a$ is elliptic, because its translation length satisfies $|p| \ell(a)=|q| \ell(a)$.

Lemma 2.1 (Forester [11]) Let $T$ be a GBS tree, with $G$ non-elementary. Any two non-trivial elliptic elements $g, g^{\prime}$ are commensurable. An element $g \in G$ is elliptic if and only if its commensurator equals $G$.

Proof If $g, g^{\prime}$ fix vertices $v, v^{\prime}$, one shows that they are commensurable by induction on the distance between $v$ and $v^{\prime}$. If $g$ is hyperbolic, its axis is $\operatorname{Comm}(g)$-invariant, so $\operatorname{Comm}(g) \neq G$ because $G$ is not elementary.

Corollary 2.2 The set of elliptic elements depends only on $G$, not on the GBS tree $T$. It is invariant under automorphisms of $G$.

As any two GBS trees have the same elliptic subgroups, Forester's deformation theorem [11] yields the following.

Corollary 2.3 Let $G$ be a non-elementary GBS group. Any two GBS trees are related (among GBS trees) by a finite sequence of elementary expansions and collapses. 
The quotient $G / G_{\text {ell }}$ of $G$ by the subgroup generated by all elliptic elements may be identified with the (topological) fundamental group $\pi_{1}^{\text {top }}(\Gamma)$ of the graph $\Gamma$ (this is a general property of graphs of groups). All labelled graphs representing $G$ thus have the same first Betti number, denoted by $b$.

All homomorphisms from $G$ to a free group with non-abelian image factor through the quotient map $\theta: G \rightarrow G / G_{\text {ell }} \simeq F_{b}$ (because in a free group the commensurator of any non-trivial element is cyclic). Since $G$ always maps onto $\mathbb{Z}$, the maximum rank of a free quotient of $G$ is $\max (b, 1)$.

\section{The modular homomorphism $\Delta$}

Let $G$ be a non-elementary GBS group. The set $\mathcal{E}$ consisting of all non-trivial elliptic elements is stable under conjugation, elements of $\mathcal{E}$ have infinite order, and any two elements of $\mathcal{E}$ are commensurable. These properties yield a homomorphism $\Delta$ from $G$ to the multiplicative group of non-zero rationals $\mathbb{Q}^{*}$, defined as follows.

Given $g \in G$, choose any $a \in \mathcal{E}$. There is a relation $g a^{p} g^{-1}=a^{q}$, with $p, q$ nonzero, and we define $\Delta(g)=\frac{p}{q}$. As pointed out in (Kropholler [19]), it is easily checked that this is independent of the choices made ( $a$ and the relation), and defines a homomorphism. We call $\Delta(g)$ the modulus of $g$. Note that $\Delta \circ \alpha=\Delta$ if $\alpha$ is any automorphism of $G$, because $\mathcal{E}$ is $\alpha$-invariant.

Let $H$ be a finite index subgroup of $G$. Any GBS $G$-tree is also a GBS $H$-tree, so $H$ is a GBS group. The modular homomorphism of $H$ is the restriction of that of $G$.

Every elliptic element has modulus 1 , so $\Delta$ factors through the free group $G / G_{\text {ell }} \simeq F_{b}$. In particular, $\Delta$ is trivial when $\Gamma$ is a tree. If $\Gamma$ is a labelled graph representing $G$, one has $G / G_{\text {ell }} \simeq \pi_{1}^{\text {top }}(\Gamma)$ and the modulus may be computed as follows (see Bass-Kulkarni [1] and Forester [13]): if $\gamma \in \pi_{1}^{\text {top }}(\Gamma)$ is represented by an edge-loop $\left(e_{1}, \ldots, e_{m}\right)$, its modulus is simply

$$
\prod_{j=1}^{m} \frac{\lambda e_{j}}{\lambda \bar{e}_{j}} .
$$

We denote by $M$ the image of $\Delta$. It is a subgroup of $\left(\mathbb{Q}^{*}, \times\right)$. If $G=B S(m, n)$, then $M$ is generated by $\frac{m}{n}$. If $G$ is represented by the labelled graph of Figure 6, then $M$ is generated by -1 and $-\frac{3}{2}$.

Remark Here is another way of viewing the modular homomorphism $\Delta$. Let $T$ be a GBS tree, and $G_{v}$ a vertex stabilizer. Since $G_{v}$ is commensurable to all its conjugates, the action of $G$ on itself by conjugation induces a homomorphism $\Delta$ from $G$ to the abstract commensurator of $G_{v}$, which is canonically isomorphic to $\mathbb{Q}^{*}$ (from this point of view, it might be better to define $\Delta(g)$ as $\frac{q}{p}$ rather than $\left.\frac{p}{q}\right)$. 
Lemma 2.4 Let $r=\frac{p}{q}$ be a non-zero rational number, written in lowest terms. Assume $r \neq \pm 1$.

(1) $r \in M$ if and only if the equation $x y^{p} x^{-1}=y^{q}$ has a solution with $y \neq 1$.

(2) If $r \in \mathbb{Z}$, then $r \in M$ if and only if $G$ contains a subgroup isomorphic to $B S(1, r)$.

Proof If $\frac{p}{q} \in M$, the equation $x y^{n p} x^{-1}=y^{n q}$ has a non-trivial solution for some $n \in \mathbb{Z}$, so $x y^{p} x^{-1}=y^{q}$ has a non-trivial solution. Conversely, if $|p| \neq|q|$ and $x y^{p} x^{-1}=y^{q}$ has a non-trivial solution, then $y$ must be elliptic and therefore $\frac{p}{q}=$ $\Delta(x) \in M$. We have proved (1).

If $x y^{r} x^{-1}=y$ with $y \neq 1$ and $r$ an integer different from $-1,0,1$, then $H=\langle x, y\rangle$ is a solvable GBS group, and $\Delta(x)=r$, so $H \simeq B S(1, r)$ (one may also show $H \simeq B S(1, r)$ by arguing that the only torsion-free proper quotient of $B S(1, r)$ is $\mathbb{Z})$.

\section{Remarks}

- The values \pm 1 are special. If $G$ is represented by a labelled tree $\Gamma$ containing a $(2,2)$-edge, then $x y x^{-1}=y^{-1}$ has a non-trivial solution because $G$ contains a Klein bottle group, but $-1 \notin M$ because $\Gamma$ is a tree. Conversely, 1 always belong to $M$, but $B S(1, n)$ does not contain $\mathbb{Z}^{2}=B S(1,1)$ for $|n|>1$.

- It is probably not true that $G$ always contains $B S(p, q)$ if $\frac{p}{q} \neq \pm 1$ is a modulus.

- Using $\Delta$, it is easy to show that the isomorphism type of $B S(m, n)$ determines $m$ and $n$ (normalized by $1 \leq m \leq|n|$ ) see Moldavanskiǔ [27]. In most cases, $m$ and $n$ are determined by $m / n$ (given by $\Delta$ ) and $|m-n|$ (given by abelianizing). To obtain $m$ from the isomorphism type of $B S(m, m)$, observe that the quotient of $B S(m, m)$ by its center is $\mathbb{Z} * \mathbb{Z} / m \mathbb{Z}$.

We say that $G$ has trivial modulus if $M=\{1\}$ (we often write this as $\Delta=1$ ). It is unimodular if $M \subset\{1,-1\}$, equivalently if $x y^{p} x^{-1}=y^{q}$ with $y \neq 1$ implies $p= \pm q$. As in [13], we say that $G$ has no non-trivial integral modulus if $M \cap \mathbb{Z} \subset\{1,-1\}$. This is equivalent to saying that $G$ contains no solvable Baumslag-Solitar group $B S(1, n)$ with $n \geq 2$ (we may take $n>0$ because $B S(1,-n)$ contains $B S\left(1, n^{2}\right)$ ).

\section{Unimodular groups}

Proposition 2.5 Let $G$ be a non-elementary GBS group. The center $Z(G)$ of $G$ is infinite cyclic if $G$ has trivial modulus, trivial otherwise. It acts as the identity on any GBS tree. 
Proof Let $T$ be any GBS tree (recall that $T$ is always assumed to be minimal). If $a$ is central (more generally, if $\langle a\rangle$ is normal), it is elliptic, as otherwise its axis would be a $G$-invariant line. The fixed point set of $a$ is a $G$-invariant subtree, so equals $T$ by minimality. This shows that $Z(G)$ is contained in the kernel of the action (elements acting on $T$ as the identity). In particular, it is trivial or cyclic.

If $\Delta(g) \neq 1$ and $a \in \mathcal{E}$, there is a relation $g a^{p} g^{-1}=a^{q}$ with $p \neq q$, so $a$ cannot be central. This shows that $Z(G)$ is trivial if $G$ does not have trivial modulus. If $\Delta$ is trivial, choose any finite generating system $s_{i}$ for $G$, and $a \in \mathcal{E}$. For each $i$, there is a relation $s_{i} a^{n_{i}} s_{i}^{-1}=a^{n_{i}}$ with $n_{i} \neq 0$. It follows that some power of $a$ is central.

Proposition 2.6 Let $G$ be a non-elementary GBS group. The following are equivalent:

(1) $G$ is unimodular.

(2) $G$ contains a normal infinite cyclic subgroup $Z$.

(3) $G$ has a finite index subgroup isomorphic to $F_{n} \times \mathbb{Z}$ for some $n>1$.

The quotient of $G$ by any normal infinite cyclic subgroup $Z$ is virtually free.

Proof Suppose $G$ is unimodular. The kernel of $\Delta$ has index 1 or 2 , and has trivial modulus. Its center is infinite cyclic and characteristic in $G$, so (1) implies (2).

Suppose $Z$ is infinite cyclic and normal. Let $T$ be any GBS tree. As in the proof of Proposition 2.5, one shows that $Z$ is contained in the kernel of the action. The quotient $G / Z$ acts on $T$ with finite stabilizers, so is virtually free. This easily implies that $G$ is virtually $F_{n} \times \mathbb{Z}$.

If $G$ is virtually $F_{n} \times \mathbb{Z}$, its modulus is trivial on a finite index subgroup, so $M$ is finite, hence contained in $\{1,-1\}$.

\section{Remarks}

- Let $T$ be a GBS tree. If $G$ is unimodular, we have seen that $Z(\operatorname{ker}(\Delta))$ is contained in the kernel of the action on $T$. Conversely, if the action has a non-trivial kernel $K$, then $G$ is unimodular (because $K$ is normal and cyclic), and furthermore $K=Z(\operatorname{ker}(\Delta))$. To see this, simply note that, if $a$ generates $K$, one has $g a g^{-1}=a^{ \pm 1}$ for any $g \in G$, so $a$ commutes with $\operatorname{ker}(\Delta)$.

- Two non-solvable GBS groups are quasi-isometric if and only if they are both unimodular or both non-unimodular [34]. Any torsion-free group quasi-isometric to $F_{n} \times \mathbb{Z}$ with $n>1$ is a unimodular GBS group [28]. 


\section{Twisted conjugacy classes}

Let $\alpha: G \rightarrow G$ be an endomorphism. Two elements $g, g^{\prime} \in G$ are $\alpha$-conjugate if there exists $h$ such that $g^{\prime}=h g \alpha(h)^{-1}$. The number of $\alpha$-conjugacy classes is the Reidemeister number of $\alpha$, denoted by $R(\alpha)$. It is relevant for fixed point theory (see Fel'shtyn-Goncalves [10]).

Proposition 2.7 Let $\alpha$ : $G \rightarrow G$ be an endomorphism of a non-elementary GBS group. If one of the following conditions holds, then $R(\alpha)$ is infinite:

(1) $\alpha$ is surjective.

(2) $\alpha$ is injective and $G$ is not unimodular.

(3) $G=B S(m, n)$ with $|m| \neq|n|$, and the image of $\alpha$ is not cyclic.

This generalizes results of [10] about Baumslag-Solitar groups.

Proof First suppose that $G$ is unimodular and $\alpha$ is surjective. The group $G$ is residually finite (because it is virtually $F_{n} \times \mathbb{Z}$ ), hence Hopfian. We therefore assume that $\alpha$ is an automorphism. The subgroup $Z=Z(\operatorname{ker}(\Delta))$ is characteristic, so $\alpha$ induces an automorphism $\beta$ on the virtually free group $G / Z$. As $G / Z$ is a nonelementary (word) hyperbolic group, $R(\beta)$ is infinite (Levitt-Lustig [24], Fel'shtyn [9]). This implies that $R(\alpha)$ is infinite.

From now on, we assume that $G$ is not unimodular. If $\alpha$ is an automorphism, we know that $\Delta \circ \alpha=\Delta$, so $\alpha$-conjugate elements of $G$ have the same modulus. As $M$ is infinite, we get $R(\alpha)$ infinite. This argument works in the general case, but we have to prove $\Delta \circ \alpha=\Delta$ for endomorphisms satisfying (1), (2), or (3).

We first claim that $\alpha$ does not factor through $\theta: G \rightarrow G / G_{\text {ell }} \simeq F_{b}$. This is clear if (2) or (3) holds. If a surjective $\alpha$ factors as $\rho \circ \theta$, then $\theta \circ \rho$ is a non-injective epimorphism from $F_{b}$ to itself, a contradiction because free groups are Hopfian.

We can now show $\Delta \circ \alpha=\Delta$. Since $\alpha$ does not factor through $\theta$, there is an elliptic $a$ with $\alpha(a) \neq 1$. As $G$ is not unimodular, there is a relation $g_{0} a^{m} g_{0}^{-1}=a^{n}$ with $|m| \neq|n|$. From $\alpha\left(g_{0}\right) \alpha(a)^{m} \alpha\left(g_{0}\right)^{-1}=\alpha(a)^{n}$, we deduce that $\alpha(a)$ is elliptic. Thus $\alpha(a)$ is a non-trivial elliptic element, and may be used to compute $\Delta$. Given any $g$, we have a relation $g a^{p} g^{-1}=a^{q}$. We then write $\alpha(g) \alpha(a)^{p} \alpha(g)^{-1}=\alpha(a)^{q}$, showing that $g$ and $\alpha(g)$ have the same modulus $\frac{p}{q}$. 


\section{The automorphism group of a GBS tree}

\section{General facts}

Let $G$ be any finitely generated group. As above, we consider $G$-trees up to equivariant isomorphism. There is a natural action of $\operatorname{Out}(G)$ on the set of $G$-trees, given by precomposing an action of $G$ on $T$ with an automorphism of $G$ (composing with an inner automorphism does not change the tree).

Given $T$, we denote by $\operatorname{Out}^{T}(G) \subset \operatorname{Out}(G)$ its stabilizer: $\Phi$ is in $\operatorname{Out}^{T}(G)$ if and only if $T$, with the action of $G$ twisted by $\Phi$, is equivariantly isomorphic to $T$ with the original action. When $T$ is irreducible, this is equivalent to saying that the length function $\ell$ satisfies $\ell \circ \Phi=\ell$.

We recall results of Levitt [22] about $\operatorname{Out}^{T}(G)$. We assume that $T$ is minimal and is not a line (but there is no condition on edge and vertex stabilizers in this subsection). The quotient graph of groups is denoted by $\Gamma$, its vertex set by $V$.

There is a natural homomorphism from $\operatorname{Out}^{T}(G)$ to the symmetry group of $\Gamma$ (viewed as a graph with no additional structure). The kernel is a finite index $\operatorname{subgroup} \operatorname{Out}_{0}^{T}(G)$, and there is a homomorphism $\rho: \operatorname{Out}_{0}^{T}(G) \rightarrow \prod_{v \in V} \operatorname{Out}\left(G_{v}\right)$. All automorphisms of $G_{v}$ which occur in the image of $\rho$ preserve the set of conjugacy classes of incident edge groups.

The kernel of $\rho$ is generated by the group of twists $\mathcal{T}(T)$ together with automorphisms called bitwists (bitwists belong to $\mathcal{T}(T)$ when vertex groups are abelian). The group $\mathcal{T}(T)$, which we also denote by $\mathcal{T}(\Gamma)$, will play an important role in the sequel. Before defining it, we mention that, when edge groups are cyclic, there is a further finite index subgroup $\operatorname{Out}_{1}^{T}(G) \subset \operatorname{Out}_{0}^{T}(G)$ with $\operatorname{Out}_{1}^{T}(G) \cap \operatorname{ker} \rho=\mathcal{T}(T)$. It will be used in Section 7.

To define $\mathcal{T}(T)$, we first consider an oriented edge $e$ of $\Gamma$, with origin $o(e)=v$. Let $G_{e}, G_{v}$ be the corresponding edge and vertex groups, with $G_{e}$ identified to its image in $G_{v}$. We denote by $Z_{G_{v}}\left(G_{e}\right)$ the centralizer of $G_{e}$ in $G_{v}$.

Given $z \in Z_{G_{v}}\left(G_{e}\right)$, we define the twist $D(z) \in \operatorname{Out}(G)$ by $z$ around $e$ as follows (see [22] for details). If $e$ is separating, it expresses $G$ as an amalgam $G=G_{1} * G_{e} G_{2}$. Then $D(z)$ is defined as the identity on $G_{1}$, and conjugation by $z$ on $G_{2}$. If $e$ does not separate, $G$ is an HNN-extension and $D(z)$ maps the stable letter $t$ to $z t$ (keeping the base group fixed).

The group of twists $\mathcal{T}(T)$, or $\mathcal{T}(\Gamma)$, is the subgroup of $\operatorname{Out}(G)$ generated by all twists. As twists around distinct edges commute, $\mathcal{T}(\Gamma)$ is a quotient of $\prod Z_{G_{o(e)}}\left(G_{e}\right)$, the 
product being taken over all oriented edges of $\Gamma$. [22, Proposition 3.1] says that only two types of relations are needed to obtain a presentation of $\mathcal{T}(\Gamma)$.

For each pair of opposite edges $(e, \bar{e})$, there are edge relations associated to elements $z$ in the center $Z\left(G_{e}\right)$ (twisting by $z$ near the origin of $e$ defines the same outer automorphism as twisting by $z^{-1}$ near the origin of $\bar{e}$ ). For each vertex $v$, there are vertex relations associated to elements $z \in Z\left(G_{v}\right)$ (twisting by $z$ simultaneously around all edges with origin $v$ defines an inner automorphism).

Remark 3.1 Let $e \subset \Gamma$ be a segment such that both adjacent vertex groups are abelian. Using the vertex relations, one sees that $\mathcal{T}(\Gamma)$ is generated by the groups $Z_{G_{o(f)}}\left(G_{f}\right)$ with $f \neq e, \bar{e}$. Collapsing $e$ yields a new graph of groups whose group of twists contains $\mathcal{T}(\Gamma)$.

Our main tool for finding free groups $F_{2}$ in $\operatorname{Out}(G)$ will be the following Lemma.

Lemma 3.2 Let $\Gamma$ be a minimal graph of groups, with fundamental group $G$. Let $e$ be an edge with origin $v$, and let $G_{e}, G_{v}$ be the corresponding groups. The subgroup $\mathcal{T}(\Gamma) \subset \operatorname{Out}(G)$ maps onto $Z_{G_{v}}\left(G_{e}\right) /\left\langle Z\left(G_{v}\right), Z\left(G_{e}\right)\right\rangle$.

We denote by $\left\langle Z\left(G_{v}\right), Z\left(G_{e}\right)\right\rangle$ the (obviously normal) subgroup generated by the centers of $G_{v}$ and $G_{e}$.

Proof Divide $\mathcal{T}(\Gamma)$ by (the image of) all factors $Z_{G_{o(f)}}\left(G_{f}\right)$ for $f \neq e$ (including $f=\bar{e})$. The only relations which remain are those involving $Z_{G_{v}}\left(G_{e}\right)$, namely edge relations associated to $(e, \bar{e})$ and vertex relations associated to $v$. The quotient is precisely $Z_{G_{v}}\left(G_{e}\right) /\left\langle Z\left(G_{v}\right), Z\left(G_{e}\right)\right\rangle$.

\section{The group of twists of a GBS tree}

Now let $G$ be a non-elementary GBS group. We consider the action of $\operatorname{Out}(G)$ on the set of GBS trees. The corresponding action on the set of marked graphs is by changing the marking. If $T$ is rigid, then $\operatorname{Out}^{T}(G)=\operatorname{Out}(G)$.

The group of twists $\mathcal{T}(T)$ is a finitely generated abelian group. The presentation recalled above may be rephrased as follows (we use additive notation).

Given an oriented edge $e$ of $\Gamma$, there is one generator $D_{e}$. If $e$ is separating, $D_{e}$ is the identity on $G_{1}$, and conjugation by $x_{v}$ on $G_{2}$ (with $x_{v}$ the generator of the vertex group at $v=o(e)$, and $G=G_{1} *_{G_{e}} G_{2}$ as above). If $e$ does not separate, choose a maximal tree $\Gamma_{0}$ not containing $e$. In the corresponding presentation of $G$, define $D_{e}$ as mapping $t_{e}$ to $x_{o(e)} t_{e}$ and keeping all other generators fixed. 
In terms of these generators $D_{e}$, the relations are the following. For each pair of opposite edges $(e, \bar{e})$, there is an edge relation $\lambda_{e} D_{e}+\lambda_{\bar{e}} D_{\bar{e}}=0$, implied by the relation $\left(x_{o(e)}\right)^{\lambda(e)}=\left(x_{o(\bar{e})}\right)^{\lambda(\bar{e})}$ or $t_{e}\left(x_{o(e)}\right)^{\lambda(e)} t_{e}^{-1}=\left(x_{o(\bar{e}}\right)^{\lambda(\bar{e})}$. For each vertex $v$, there is a vertex relation $\sum_{e \in E_{v}} D_{e}=0$, with $E_{v}$ the set of edges with origin $v$.

Remark Recall that $G$ is in a natural way the fundamental group of a 2-complex consisting of annuli glued to circles. One can consider the subgroup $D T(\Gamma)$ of $\mathcal{T}(\Gamma)$ generated by Dehn twists supported in the annuli. It is easy to see that it has finite index. One may also show $\mathcal{T}(\Gamma)=D T\left(\Gamma^{\prime}\right)$, where $\Gamma^{\prime}$ is a (non reduced) graph obtained from $\Gamma$ by elementary expansions.

Recall that $b$ is the first Betti number of any labelled graph $\Gamma$ representing $G$.

Proposition 3.3 Let $G$ be a non-elementary GBS group. Define $k$ as $b$ if $G$ has trivial modulus, $b-1$ if not.

(1) The torsion-free $\operatorname{rank} \operatorname{rk}\left(G_{a b}\right)$ of the abelianization $G_{a b}$ is $k+1$.

(2) Let $\Gamma$ be any labelled graph representing $G$. The torsion-free rank of the abelian group $\mathcal{T}(\Gamma)$ is $k$.

(3) If $\Gamma_{0} \subset \Gamma$ is a maximal subtree, the twists $D_{e}$ around the edges of $\Gamma \backslash \Gamma_{0}$ generate a finite index subgroup of $\mathcal{T}(\Gamma)$.

Proof Killing all elliptic elements produces an epimorphism $\theta: G \rightarrow F_{b}$ (see Section 2 ), so $\operatorname{rk}\left(G_{a b}\right) \geq b$. If $\Delta$ is non-trivial, any elliptic element $a$ satisfies a relation $g a^{p} g^{-1}=a^{q}$ with $p \neq q$, so is mapped trivially to torsion-free abelian groups. This shows $\operatorname{rk}\left(G_{a b}\right)=b$ in this case.

If $\Delta$ is trivial, fix $\Gamma$ and $\Gamma_{0}$. It follows from the presentation of $G$ given earlier that $G_{a b}$ is the direct sum of $\mathbb{Z}^{b}$ with the abelian group $G^{\prime}$ defined by the following presentation: there is one generator $x_{v}$ for each vertex of $\Gamma$, and one relation $\lambda_{e} x_{o(e)}=\lambda_{\bar{e}} x_{o(\bar{e})}$ for each pair of opposite edges $(e, \bar{e})$. We show that $G^{\prime}$ maps non-trivially to $\mathbb{Z}$. It is easy to map the generators $x_{v}$ to $\mathbb{Z}$ in such a way that relations associated to edges in $\Gamma_{0}$ are satisfied. Using the formula

$$
\Delta(\gamma)=\prod_{j=1}^{m} \frac{\lambda_{e_{j}}}{\lambda_{\bar{e}_{j}}}
$$

(see Section 2), one sees that the remaining relations are automatically satisfied. We get $\operatorname{rk}\left(G_{a b}\right)=b+1$. 
Assertion (2) follows immediately from Proposition 2.5 and the exact sequence

$$
0 \rightarrow Z(G) \rightarrow \mathbb{Z}^{v+\zeta} \rightarrow \mathbb{Z}^{2 \zeta} \rightarrow \mathcal{T} \rightarrow 0
$$

given by [22, Proposition 3.1], where $v$ (resp. $\zeta$ ) is the number of vertices (resp. edges) of $\Gamma$ (we are grateful to M Clay for suggesting this short argument).

Assertion (3) follows from the presentation of $\mathcal{T}(\Gamma)$ in terms of the generators $D_{e}$. If we add the relations $D_{e}=D_{\bar{e}}=0$ for $e \notin \Gamma_{0}$, the quotient is the group of twists associated to the labelled graph $\Gamma_{0}$, so is finite by Assertion (2).

Remark 3.4 One may decide whether a given $D_{e}$ has finite or infinite order. View $\Delta$ as a map defined on $\pi_{1}^{\text {top }}(\Gamma)$. If $e$ does not separate, the order of $D_{e}$ is finite if and only if $G$ has non-trivial modulus, but every curve not containing $e$ has trivial modulus. If $e$ separates, the order is infinite if and only if each component of $\Gamma \backslash\{e\}$ contains a curve with non-trivial modulus.

The groups $\mathcal{T}(T)$ associated to different GBS trees are abstractly commensurable by Proposition 3.3. We show that they are commensurable as subgroups of $\operatorname{Out}(G)$.

Proposition 3.5 If $T, T^{\prime}$ are two GBS trees, then $\mathcal{T}(T)$ and $\mathcal{T}\left(T^{\prime}\right)$ are commensurable subgroups of $\operatorname{Out}(G)$.

Proof By Corollary 2.3, it suffices to show that $\mathcal{T}\left(T^{\prime}\right)$ is commensurable with $\mathcal{T}(T)$ if $T^{\prime}$ is obtained from $T$ by an elementary collapse. Consider the corresponding graphs $\Gamma, \Gamma^{\prime}$. Let $e=v w \subset \Gamma$ be the collapsed edge. We assume $\lambda_{e}=1$, and we denote $\lambda_{\bar{e}}$ by $\lambda$, so $G_{v}$ has index $\lambda$ in $G_{w}$.

Let $F$ be the set of oriented edges of $\Gamma$ other than $e, \bar{e}$. The group $\mathcal{T}(T)$ is the subgroup of $\operatorname{Out}(G)$ generated by the twists $D_{f}, f \in F$ (see Remark 3.1). Similarly, $\mathcal{T}\left(T^{\prime}\right)=\left\langle D_{f}^{\prime} \mid f \in F\right\rangle$, as $F$ may be viewed as the set of oriented edges of $\Gamma^{\prime}$. Moreover, we have $D_{f}=D_{f}^{\prime}$ if the origin of $f$ is not $v$, and $D_{f}=\lambda D_{f}^{\prime}$ if it is because the collapse replaces the vertex group $G_{v}$ of $\Gamma$ by the larger group $G_{w}$. This shows that $\mathcal{T}\left(T^{\prime}\right)$ contains $\mathcal{T}(T)$ as a subgroup of finite index.

Remark 3.6 The index of $\mathcal{T}(T)$ in $\mathcal{T}\left(T^{\prime}\right)$ divides a power of the label $\lambda$. This will be used in the proof of Theorem 3.12. 


\section{Applications}

We apply the preceding results to the study of $\operatorname{Out}^{T}(G)$, using the following fact.

Proposition 3.7 (Levitt [22]) $\mathcal{T}(T)$ has finite index in $\operatorname{Out}^{T}(G)$.

This follows from [22, Theorem 1.6], as edge and vertex groups have finite outer automorphism groups. More precisely, let us show the following Proposition.

Proposition 3.8 Given $G$, the index of $\mathcal{T}(T)$ in $\mathrm{Out}^{T}(G)$ is uniformly bounded (independently of $T$ ).

Proof Consider the chain of subgroups $\mathcal{T}(T) \subset \operatorname{ker} \rho \subset \mathrm{Out}_{0}^{T}(G) \subset \mathrm{Out}^{T}(G)$ mentioned at the beginning of this section. We check that each group has uniformly bounded index in the next.

The index of $\operatorname{Out}_{0}^{T}(G)$ in $\operatorname{Out}^{T}(G)$ is bounded by the order of the symmetry group of $\Gamma$. The number of edges of $\Gamma$ is not always uniformly bounded, but the first Betti number is fixed, and there is a uniform bound for the number $d$ of terminal vertices (because adding the relations $x_{v}=1$, for $v$ non-terminal, maps $G$ onto the free product of $d$ non-trivial finite cyclic groups). This is enough to bound the symmetry group.

The map $\rho$ describes how automorphisms act on vertex groups. Since these groups are all commensurable, and isomorphic to $\mathbb{Z}$, the image of $\rho$ has order at most 2 , so $\operatorname{ker} \rho$ has index at most 2 in $\operatorname{Out}_{0}^{T}(G)$. Finally, $\operatorname{ker} \rho$ is generated by $\mathcal{T}(T)$ together with bitwists. As vertex groups are abelian, bitwists belong to $\mathcal{T}(T)$, so $\mathcal{T}(T)=\operatorname{ker} \rho$.

If $T$ is rigid, then $\operatorname{Out}^{T}(G)=\operatorname{Out}(G)$. We get the following Theorem.

Theorem 3.9 If $G$ is algebraically rigid, then $\operatorname{Out}(G)$ contains $\mathbb{Z}^{k}$ as a subgroup of finite index.

In general, we have the following Theorem.

Theorem 3.10 Up to commensurability, the subgroup $\operatorname{Out}^{T}(G)$ of $\operatorname{Out}(G)$ does not depend on $T$. It contains $\mathbb{Z}^{k}$ with finite index.

Another proof of the first assertion (and therefore of Proposition 3.5, but not of Remark 3.6) is given in Section 5. Also note the following related result.

Theorem 3.11 (Clay [5]) Any subgroup of $\operatorname{Out}(G)$ commensurable with a subgroup of $\mathrm{Out}^{T}(G)$ is contained in $\mathrm{Out}^{T^{\prime}}(G)$ for some GBS tree $T^{\prime}$. 
We now prove the following Theorem.

Theorem 3.12 The set of prime numbers $p$ such that $\operatorname{Out}(G)$ contains non-trivial $p$-torsion is finite.

Proof As any torsion element of $\operatorname{Out}(G)$ is contained in some $\operatorname{Out}^{T}(G)$ by [5], and the index of $\mathcal{T}(T)$ in $\operatorname{Out}^{T}(G)$ is uniformly bounded by Proposition 3.8, it suffices to control torsion in groups of twists.

First note that the set of prime numbers dividing a label of $\Gamma$ does not depend on $\Gamma$, as it does not change during an elementary collapse. Call it $\mathcal{P}$. If $T$ and $T^{\prime}$ are related by an elementary collapse, Remark 3.6 shows that $\mathcal{T}(T)$ and $\mathcal{T}\left(T^{\prime}\right)$ have torsion at the same primes, except possibly those in $\mathcal{P}$. This implies that only finitely many primes may appear in the torsion of a group of twists: those in $\mathcal{P}$, and those in the torsion of $\mathcal{T}\left(T_{0}\right)$ for some fixed $T_{0}$.

We have seen that $\operatorname{Out}(B S(2,4))$ contains arbitrarily large 2-torsion.

The proof of Theorem 3.12 also shows the following Corollary.

Corollary 3.13 If $p$ is a prime number such that $\operatorname{Out}(G)$ contains $p$-torsion of arbitrarily large order, then $p$ divides at least one label of each labelled graph representing $G$.

We do not know whether $p$ must divide some integral modulus.

\section{Unimodular groups}

Let $\Gamma$ be a labelled graph representing a non-elementary unimodular group $G$, and $T$ the associated Bass-Serre tree. We denote by $G^{+}$the kernel of $\Delta: G \rightarrow\{ \pm 1\}$ (positive elements). All elliptic elements are positive. Let $Z$ be the center of $G^{+}$. We know that it is cyclic, characteristic in $G$, and acts as the identity on $T$.

We fix a non-trivial $\delta \in Z$. If $\Delta=1$ we may take $\delta$ to be a generator $\delta_{0}$, but the study of $\operatorname{Out}(G)$ when $\Delta \neq 1$ will require $\delta$ to be $\left(\delta_{0}\right)^{4}$. Note that any generator of an edge or vertex group is a root of $\delta$.

Let $Z^{\prime}$ be the cyclic group generated by $\delta$. There is an exact sequence $\{1\} \rightarrow Z^{\prime} \rightarrow$ $G \rightarrow H \rightarrow\{1\}$ with $H$ virtually free. The group $H$ is the fundamental group of a graph of groups with the same underlying graph. Vertex and edge groups are finite 
cyclic groups, the order being the index of $\langle\delta\rangle$ in the original group. We denote by $\bar{g}$ the image of $g \in G$ in $H$.

Since $Z^{\prime}$ is characteristic in $G$, there are natural homomorphisms $\operatorname{Aut}(G) \rightarrow \operatorname{Aut}(H)$ and $\operatorname{Out}(G) \rightarrow \operatorname{Out}(H)$. The basic example is $\operatorname{Out}\left(F_{n} \times \mathbb{Z}\right)$, which contains the semidirect product $\mathbb{Z}^{n} \rtimes \operatorname{Out}\left(F_{n}\right)$ with index 2 (the factor $\mathbb{Z}^{n}$ should be thought of as $\left.\operatorname{Hom}\left(F_{n}, \mathbb{Z}\right)\right)$. But the following examples illustrate a few of the subtleties involved when trying to lift automorphisms from $H$ to $G$.

\section{Examples}

- Let $G$ be $\left\langle a, b \mid a^{3}=b^{3}\right\rangle$ and $H$ be $\left\langle\bar{a}, \bar{b} \mid \bar{a}^{3}=\bar{b}^{3}=1\right\rangle$. The automorphism of $H$ mapping $\bar{a}$ to $\bar{a}^{-1}$ and $\bar{b}$ to $\bar{b}$ does not lift to $G$.

- $G$ is $B S(3,3)=\left\langle a, t \mid t a^{3} t^{-1}=a^{3}\right\rangle$ and $H$ is $\left\langle\bar{a}, \bar{t} \mid \bar{a}^{3}=1\right\rangle$. The automorphism fixing $\bar{a}$ and sending $\bar{t}$ to $\bar{t} \bar{a}$ has order 3 , but all its lifts have infinite order.

- $G$ is $B S(2,-2)=\left\langle a, t \mid t a^{2} t^{-1}=a^{-2}\right\rangle$ and $H$ is $\left\langle\bar{a}, \bar{t} \mid \bar{a}^{2}=1\right\rangle$. Conjugation by $\bar{a}$ in $H$ has lifts of order 2 , such as $a \mapsto a, t \mapsto a t a$, or $a \mapsto a^{-1}, t \mapsto a t a^{-1}$, but no lift of order 2 is inner.

Let $H^{+}$be the image of $G^{+}$in $H$. If $\Delta \neq 1$, it has index 2 (because $\delta$ is positive). There are only finitely many conjugacy classes of torsion elements in $H$ (they all come from vertex groups). All torsion elements of $H$ belong to $H^{+}$, but a conjugacy class in $H$ may split into two classes in $H^{+}$.

We shall now define a homomorphism $\tau: G \rightarrow \operatorname{Isom}(\mathbb{R})$ (it is similar to the homomorphism $G^{\prime} \rightarrow \mathbb{Z}$ constructed in the proof of Proposition 3.3). We fix a maximal tree $\Gamma_{0} \subset \Gamma$. Recall the presentation of $G$ with generators $x_{v}, t_{\varepsilon}$ and relations of the form $x_{v}^{m}=x_{w}^{n}$ or $t_{\varepsilon} x_{v}^{m} t_{\varepsilon}^{-1}=x_{w}^{n}$.

To define $\tau$, send $\delta$ to $x \mapsto x+1$, send $x_{v}$ to $x \mapsto x+1 / n_{v}$ if $\delta=x_{v}^{n_{v}}$, send $t_{\varepsilon}$ to $x \mapsto \Delta\left(t_{\varepsilon}\right) x$, and check that the relations are satisfied. This $\tau$ is not canonical (it depends on the choice of $\Gamma_{0}$ ); it is uniquely defined on elliptic elements once $\delta$ has been chosen.

The image of $\tau$ in $\operatorname{Isom}(\mathbb{R})$ is infinite cyclic if $\Delta=1$, infinite dihedral if $\Delta \neq 1$. Its kernel contains no non-trivial elliptic element. The coefficient of $x$ in $\tau(g)$ is $\Delta(g)$, and $\tau\left(g c g^{-1}\right)=\tau(c)^{\Delta(g)}$ if $c$ is positive (in particular if $c$ is elliptic).

The map $\tau$ induces a map $\bar{\tau}$ from $H$ to a finite group $F$ (the quotient of the image of $\tau$ by $x \mapsto x+1)$. The group $F$ is cyclic if $\Delta=1$, dihedral if $\Delta \neq 1$.

Definition We define the finite index subgroup $\operatorname{Aut}_{0}(H) \subset \operatorname{Aut}(H)$ as the set of automorphisms $\bar{\alpha}$ such that: 
(1) $\bar{\alpha}\left(H^{+}\right)=H^{+}$.

(2) $\bar{\alpha}$ acts trivially on the set of $\mathrm{H}^{+}$-conjugacy classes of torsion elements.

(3) $\bar{\tau} \circ \bar{\alpha}=\bar{\tau}$.

Lemma 4.1 Let $\bar{\alpha} \in \operatorname{Aut}_{0}(H)$. There exists a unique lift $\alpha \in \operatorname{Aut}(G)$ such that $\tau \circ \alpha=\tau$. It satisfies $\alpha(\delta)=\delta$.

Proof Uniqueness is easy: $\alpha(g)$ is determined up to a power of $\delta$, and that power is determined by applying $\tau$.

We define $\alpha$ on the generators of $G$. In $H$, the element $\bar{x}_{v}$ has finite order and therefore is mapped by $\bar{\alpha}$ to $\bar{g}_{v} \bar{x}_{v} \bar{g}_{v}^{-1}$ for some $\bar{g}_{v} \in H^{+}$. We define $\alpha\left(x_{v}\right)=g_{v} x_{v} g_{v}^{-1}$, where $g_{v} \in G^{+}$is any lift of $\bar{g}_{v}$. Note that $\tau\left(\alpha\left(x_{v}\right)\right)=\tau\left(x_{v}\right)$ because $x_{v}$ and $g_{v}$ are positive. If $x_{v}^{m}=x_{w}^{n}$ is a relation, then $\alpha\left(x_{v}\right)^{m} \alpha\left(x_{w}\right)^{-n}$ is 1 because it is killed both in $H$ and by $\tau$. Note that $\alpha(\delta)=\alpha\left(x_{v}^{n_{v}}\right)=g_{v} x_{v}^{n_{v}} g_{v}^{-1}=\delta$.

Now consider a generator $t_{\varepsilon}$, and a lift $u_{\varepsilon}$ of $\bar{\alpha}\left(\bar{t}_{\varepsilon}\right)$. Since $\bar{\tau} \circ \bar{\alpha}=\bar{\tau}$, the elements $t_{\varepsilon}$ and $u_{\varepsilon}$ have the same image in $F$, so $\tau\left(t_{\varepsilon} u_{\varepsilon}^{-1}\right)$ is translation by an integer $n_{\varepsilon}$. We define $\alpha\left(t_{\varepsilon}\right)$ as $\delta^{n_{\varepsilon}} u_{\varepsilon}$, so that $\tau\left(\alpha\left(t_{\varepsilon}\right)\right)=\tau\left(t_{\varepsilon}\right)$. Given a relation $t_{\varepsilon} x_{v}^{m} t_{\varepsilon}^{-1}=x_{w}^{n}$, the relation $\alpha\left(t_{\varepsilon}\right) \alpha\left(x_{v}\right)^{m} \alpha\left(t_{\varepsilon}\right)^{-1}=\alpha\left(x_{w}\right)^{n}$ holds modulo $\delta$. It also holds when we apply $\tau$, so it holds in $G$.

We have constructed an endomorphism of $G$ fixing $\delta$ and inducing $\bar{\alpha}$, and this forces it to be an automorphism.

Let $\operatorname{Aut}_{0}(G) \subset \operatorname{Aut}(G)$ be the finite index subgroup consisting of automorphisms fixing $\delta$ and mapping into $\operatorname{Aut}_{0}(H)$. We know that the map $\varphi: \operatorname{Aut}_{0}(G) \rightarrow \operatorname{Aut}_{0}(H)$ is onto and has a section. We consider its kernel.

Lemma 4.2 The kernel $N$ of $\varphi: \operatorname{Aut}_{0}(G) \rightarrow \operatorname{Aut}_{0}(H)$ is isomorphic to $\mathbb{Z}^{b}$. It is generated by twists by $\delta$ around the edges of $\Gamma \backslash \Gamma_{0}$.

Recall that $b$ is the first Betti number of $\Gamma$.

Remark It is a general fact that, whenever $Z \subset G$ is characteristic, the kernel of the map $\operatorname{Aut}(G) \rightarrow \operatorname{Aut}(Z) \times \operatorname{Aut}(G / Z)$ is abelian (Raptis-Varsos [32, Proposition 2.5]). To see this, take $\alpha_{1}, \alpha_{2}$ in the kernel. Write $\alpha_{1}(g)=z_{1} g$ and $\alpha_{2}(g)=g z_{2}$ (with $z_{1}, z_{2} \in Z$, depending on $g$ ), and deduce $\alpha_{1} \alpha_{2}(g)=\alpha_{2} \alpha_{1}(g)=z_{1} g z_{2}$ (one can also prove that $z_{1}$ must be in the center of $G$ ) 
Proof Suppose $\alpha \in N$. We have $\alpha(\delta)=\delta$. If $x$ is a root of $\delta$, we have $\alpha(x)=x \delta^{p}$ and $\delta=x^{q}$, so that $\delta=\alpha\left(x^{q}\right)=\delta \delta^{p q}$ and $p=0$. Therefore $\alpha$ fixes every elliptic element. Furthermore $\alpha\left(t_{\varepsilon}\right)=\delta^{n_{\varepsilon}} t_{\varepsilon}$ for some $n_{\varepsilon} \in \mathbb{Z}$, so $\alpha$ is a product of powers of twists by $\delta$. Conversely, each choice of integers $n_{\varepsilon}$ determines an automorphism fixing all elliptic elements and belonging to $N$.

We have proved the following Theorem.

Theorem 4.3 If $G$ is non-elementary and unimodular, there is a split exact sequence

$$
\{1\} \rightarrow \mathbb{Z}^{b} \rightarrow \operatorname{Aut}_{0}(G) \stackrel{\varphi}{\rightarrow} \operatorname{Aut}_{0}(H) \rightarrow\{1\},
$$

where $H$ is virtually free and $\mathrm{Aut}_{0}$ has finite index in Aut.

We shall now show the following.

Theorem 4.4 If $G$ is non-elementary and unimodular, there is a split exact sequence

$$
\{1\} \rightarrow \mathbb{Z}^{k} \rightarrow \mathrm{Out}_{0}(G) \stackrel{\psi}{\rightarrow} \operatorname{Out}_{0}(H) \rightarrow\{1\},
$$

where $H$ is virtually free and $\mathrm{Out}_{0}$ has finite index in Out.

See Proposition 3.3 for the definition and properties of $k$.

Since $\operatorname{Out}(H)$ is VF [20], this implies the following Corollary.

Corollary 4.5 $\operatorname{Out}(G)$ and $\operatorname{Aut}(G)$ are virtually torsion-free and $V F$ (they have finite index subgroups with finite classifying spaces).

Proof of Theorem 4.4 We denote by Out ${ }_{0}$ the image of Aut ${ }_{0}$ in Out (note that Aut $_{0}$ does not contain all inner automorphisms if $\Delta \neq 1$ ), and by $\hat{N}$ the image of $N$ in $\operatorname{Out}(G)$. Let $\psi: \operatorname{Out}_{0}(G) \rightarrow \operatorname{Out}_{0}(H)$ be the natural map. Note that $\widehat{N}$ is contained in ker $\psi$, and has torsion-free rank $k$ by Lemma 4.2 and Assertion (3) of Proposition 3.3. We shall show $\operatorname{ker} \psi=\widehat{N} \simeq \mathbb{Z}^{k}$. We write $i_{g}$ for conjugation by $g$.

First assume $\Delta=1$. Then $k=b$, and $\widehat{N} \simeq \mathbb{Z}^{b}$ because it has torsion-free rank $b$ and is a quotient of $N \simeq \mathbb{Z}^{b}$. Since the image of $\tau$ is abelian, every conjugation $i_{g}$ in $G$ satisfies $\tau \circ i_{g}=\tau$, and Lemma 4.1 lifts $i_{h} \in \operatorname{Aut}_{0}(H)$ to $i_{g}$, where $g$ is any lift of $h$. Thus $\psi$ has a section.

There remains to show $\operatorname{ker} \psi \subset \hat{N}$. If $\alpha \in \operatorname{Aut}_{0}(G)$ represents an element of ker $\psi$, its image $\bar{\alpha}$ in $\operatorname{Aut}_{0}(H)$ is conjugation by some $h \in H$. Lift $i_{h}$ to $i_{g} \in \operatorname{Aut}_{0}(G)$, and 
consider $i_{g}^{-1} \alpha$. It belongs to $N$, and has the same image as $\alpha$ in $\operatorname{Out}(G)$. This implies $\operatorname{ker} \psi=\hat{N}$.

Now suppose $\Delta \neq 1$. In this case we have to choose $\delta=\left(\delta_{0}\right)^{4}$, where $\delta_{0}$ is a generator of $Z$ (the center of $G^{+}$). We first show that $\operatorname{ker} \psi=\hat{N}$. The argument is the same as before, but we have to prove that $i_{h}$ has a lift $i_{g} \in \operatorname{Aut}_{0}(G)$ (we will see that Lemma 4.1 lifts inner automorphisms to inner automorphisms, but we cannot claim it at this point). Since $\bar{\tau} \circ \bar{\alpha}=\bar{\tau}$, the image $\bar{\tau}(h)$ is central in $F$. Our choice of $\delta$ ensures that the center of $F$ has order 2 (it is generated by the image of $x \mapsto x+1 / 2$ ). In particular, $h$ is positive. If $g \in G$ is a lift of $h$, it commutes with $\delta$ and therefore $i_{g}$ belongs to $\operatorname{Aut}_{0}(G)$.

We now prove $\hat{N} \simeq \mathbb{Z}^{b-1}$. Recall that $\hat{N}$ has torsion-free rank $b-1$.

Consider the twist $D$ by $\delta=\left(\delta_{0}\right)^{4}$ around the edges $\varepsilon$ of $\Gamma \backslash \Gamma_{0}$ such that $\Delta\left(t_{\varepsilon}\right)=-1$ (it fixes the generators $x_{v}$, and maps $t_{\varepsilon}$ to $t_{\varepsilon}$ if $\Delta\left(t_{\varepsilon}\right)=1$, to $\delta t_{\varepsilon}$ if $\left.\Delta\left(t_{\varepsilon}\right)=-1\right)$. Note that $D$ is conjugation by $\left(\delta_{0}\right)^{2}$. Indeed, the $t_{\varepsilon}$ 's with modulus 1 , and the $x_{v}$ 's, are fixed by $D$ and commute with $\delta_{0}$, whereas $\left(\delta_{0}\right)^{4} t_{\varepsilon}=\left(\delta_{0}\right)^{2} t_{\varepsilon}\left(\delta_{0}\right)^{-2}$ if $t_{\varepsilon} \delta t_{\varepsilon}^{-1}=\delta^{-1}$. Since $D$ belongs to a basis of $N$ (see Lemma 4.2), the image $\hat{N}$ of $N$ in $\operatorname{Out}(G)$ is isomorphic to $\mathbb{Z}^{b-1}$.

Finally, we show that Lemma 4.1 lifts inner automorphisms to inner automorphisms (and therefore $\psi$ has a section).

Suppose $i_{h}$ belongs to $\operatorname{Aut}_{0}(H)$. Then $\bar{\tau}(h)$ is central in $F$, and therefore $\bar{\tau}\left(h^{2}\right)$ is trivial. If $g \in G$ is a lift of $h^{2}$, then $\tau(g)$ is an integral translation and we can redefine $g$ (multiplying it by a power of $\delta$ ) so that $\tau(g)$ is trivial. Then $\tau\left(g u g^{-1}\right)=\tau(u)$ for every $u \in G$, showing that Lemma 4.1 lifts conjugation by $h^{2}$ to conjugation by $g$ in $G$. Now consider the lift $\alpha$ of $i_{h}$ given by Lemma 4.1. It satisfies $\alpha^{2}=i_{g}$, and its image in $\operatorname{Out}(G)$ belongs to $\operatorname{ker} \psi$. Since $\operatorname{ker} \psi=\widehat{N}$ is torsion-free, we conclude that $\alpha$ is inner.

\section{The deformation space}

Let $G$ be a non-elementary GBS group. In this section, we work with metric GBS trees: $T$ is a metric tree, and $G$ acts by isometries. Metric trees are considered up to $G$-equivariant isometry.

Let $\mathcal{D}$ be the space of metric GBS trees, and $P \mathcal{D}$ its projectivization (obtained by identifying two trees if they differ by rescaling the metric). We call $P \mathcal{D}$ the (canonical) projectivized deformation space of $G$. 
Choosing a $G$-invariant metric on a given simplicial tree amounts to assigning a positive length to each edge of $\Gamma=T / G$. This makes $P \mathcal{D}$ into a complex. An open simplex is the set of trees with a given underlying simplicial tree, a closed simplex is the set of GBS trees that may be obtained from trees in an open simplex by elementary collapses (closed simplices have "faces at infinity", as the length of a non-collapsible edge is not allowed to be 0 ). Every closed simplex contains reduced trees.

The group $\operatorname{Out}(G)$ acts on $P \mathcal{D}$. There is a bijection between the set of orbits of open simplices and the set of (unmarked) labelled graphs representing $G$ (up to admissible sign changes). Standard techniques show that $P \mathcal{D}$ has a natural $\operatorname{Out}(G)$ equivariant deformation retraction onto a simplicial complex (see Culler-Vogtmann [8] and McCullough-Miller [26]).

GBS trees are locally finite. This implies that the complex $P \mathcal{D}$ is locally finite. Indeed, closed simplices containing $T$ consist of simplicial trees obtained from $T$ by expansion moves. Performing such moves on $T$ amounts to blowing up each vertex $v$ of $T$ into a subtree. Since $v$ has finite valence, there are only finitely many ways of expanding (not taking the metric into account). As remarked by M Clay, this local finiteness gives another proof of the first assertion of Theorem 3.10.

In general, there are several ways to define a topology on spaces of trees (equivariant Gromov-Hausdorff topology, axes topology, weak topology), but because of local finiteness they all coincide on $P \mathcal{D}$ (see the discussion in Guirardel-Levitt $[16 ; 17]$ ). Clay [4] proved that $P \mathcal{D}$ is contractible (see also [16]). By Theorem 3.10, stabilizers for the action of $\operatorname{Out}(G)$ on $P \mathcal{D}$ are virtually $\mathbb{Z}^{k}$.

This is summed up by the following Proposition.

Proposition 5.1 $\operatorname{Out}(G)$ acts on the locally finite, contractible, complex $P \mathcal{D}$ with stabilizers virtually $\mathbb{Z}^{k}$.

If $G$ is algebraically rigid, the unique reduced GBS tree belongs to every closed simplex, $P \mathcal{D}$ is a finite complex, and the action of $\operatorname{Out}(G)$ on $P \mathcal{D}$ has a fixed point. If $G$ is not algebraically rigid, we will show that $\operatorname{Out}^{T}(G)$ always has infinite index in $\operatorname{Out}(G)$ (see Theorem 8.5). All Out $(G)$-orbits are therefore infinite.

Under suitable hypotheses, we now show that $P \mathcal{D}$ is "small" and we deduce information on $\operatorname{Out}(G)$.

\section{Groups with no non-trivial integral modulus}

Suppose that $G$ has no integral modulus other than \pm 1 (equivalently, $G$ does not contain a solvable Baumslag-Solitar group $B S(1, n)$ with $n>1)$. In this case, there 
are only finitely many $\operatorname{Out}(G)$-orbits of simplices consisting of reduced trees [13, Theorem 8.2], and therefore $\operatorname{Out}(G)$ acts on the complex $P \mathcal{D}$ with only finitely many orbits. This implies the following Theorem.

Theorem 5.2 Let $G$ be a non-elementary GBS group with no integral modulus other than \pm 1 .

(1) $\operatorname{Out}(G)$ is $F_{\infty}$ (it has a $K(\pi, 1)$ with finitely many cells in every dimension).

(2) There is a bound for the cohomological dimension of torsion-free subgroups of $\operatorname{Out}(G)$.

(3) If $\operatorname{Out}(G)$ is virtually torsion-free, it has a finite index subgroup with a finite classifying space.

(4) $\operatorname{Out}(G)$ contains only finitely many conjugacy classes of finite subgroups.

Proof The first three assertions follow from Proposition 5.1 by standard techniques. Unfortunately, we do not know whether $\operatorname{Out}(G)$ must be virtually torsion-free when $G$ is not unimodular. Assertion (4) follows from Theorem 3.10 and Theorem 3.11: Any finite subgroup is contained in some $\operatorname{Out}^{T}(G)$, and it is well-known that there are finitely many conjugacy classes of finite subgroups in a group which is virtually $\mathbb{Z}^{k}$ (a proof appears in Levitt [22]).

\section{Groups with no strict ascending loop}

It is shown in [16] that, if no reduced labelled graph contains a strict ascending loop, there is an $\operatorname{Out}(G)$-equivariant deformation retraction from $P \mathcal{D}$ onto a finitedimensional subcomplex. This implies the following Theorem.

Theorem 5.3 If no reduced labelled graph representing $G$ contains a strict ascending loop, there is a bound for the cohomological dimension of torsion-free subgroups of $\operatorname{Out}(G)$.

\section{Free subgroups in $\operatorname{Out}(G)$}

Let $\Gamma$ be a labelled graph. Recall that we consider it up to admissible sign changes. In particular, when we focus on an edge, we will always assume that it is a $(p, q)$-segment with $p, q \geq 1$ ( $\geq 2$ if $\Gamma$ is reduced), or a $(p, q)$-loop with $1 \leq p \leq|q|$. Recall that a strict ascending loop is a $(1, q)$-loop with $|q| \geq 2$. A pseudo-ascending loop is a $(p, q)$-loop with $p \mid q$. 


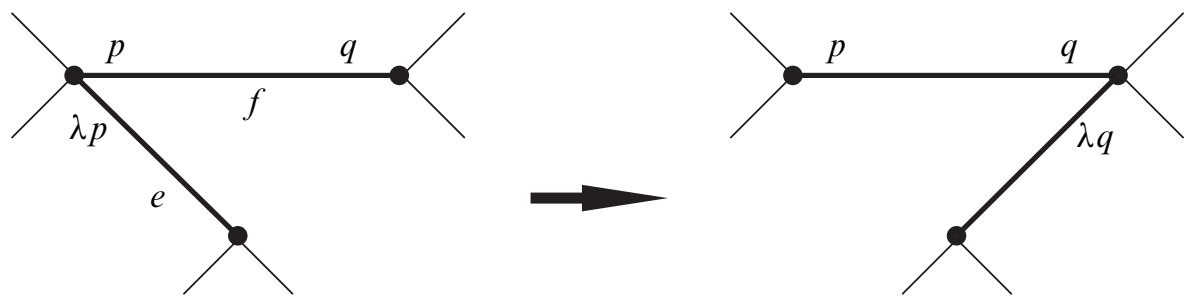

Figure 7: Slide move.

If $e, f$ are distinct oriented edges with the same origin $v$, and the label $\lambda_{f}$ of $f$ at $v$ divides the label $\lambda_{e}$ of $e$, one may slide $e$ across $f$, replacing its label by $\frac{\lambda_{e}}{\lambda_{f}} \lambda_{\bar{f}}$ (see Figure 7); both $e$ and $f$ may be loops, but they have to be distinct geometric edges $(f \neq \bar{e})$. See [13] for details about slide moves. The important thing for us here is that performing a slide move on a labelled graph gives another labelled graph representing the same group $G$ (only the GBS tree changes).

An edge $f$ is a slid edge if some other edge may slide across $f$ or $\bar{f}$ (we usually think of slid edges as non-oriented edges). For example, any $(1, q)$-loop is a slid edge if $\Gamma$ contains more than one edge (ie if $G$ is not solvable).

The goal of this section is to prove the following result (see Figure 8, where the numbers within parentheses refer to the assertions of the theorem).

Theorem 6.1 Let $\Gamma$ be a reduced labelled graph representing $G$. Suppose that $\operatorname{Out}(G)$ does not contain $F_{2}$. Then:

(1) A slid edge is either a $(2,2)$-segment or a $(1, q)-$ loop.

(2) Slid edges are disjoint.

(3) A pseudo-ascending loop is a $(p, \pm p)$-loop or a $(1, q)-$ loop.

(4) If $v$ is the basepoint of a $(1, q)$-loop with $|q| \geq 2$, then no other label at $v$ divides a power of $q$.

(5) If $v$ is the basepoint of a $(1, q)$-loop with $|q| \geq 2$, and $r, s$ are two labels at $v$ not carried by the loop, then $s$ does not divide any $r q^{n}$.

(6) Let $v w$ be a $(2,2)$-segment. Let $r$ be a label at $v$, and $s$ a label at $w$ (other than those carried by $v w$ ). If $r \mid s$ and $s$ is even, then $r=s$ and the labels are carried by the same non-oriented edge.

We prove Theorem 6.1 in several steps. Our two main tools will be slide moves and Lemma 3.2. 


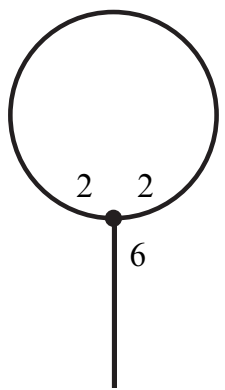

(1)

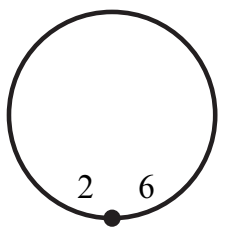

(3)

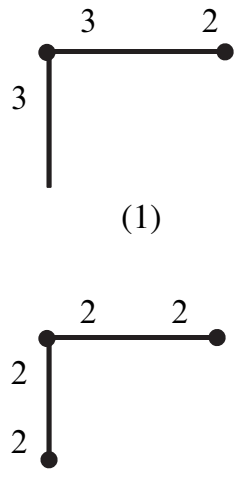

(2)

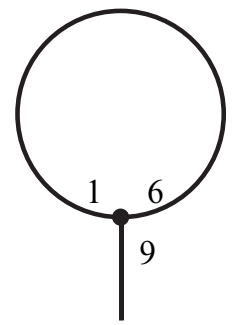

(4)

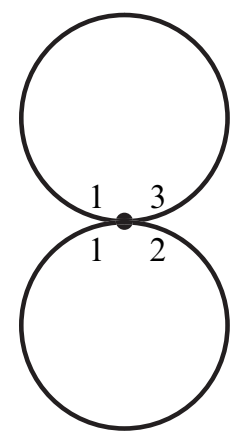

(2)

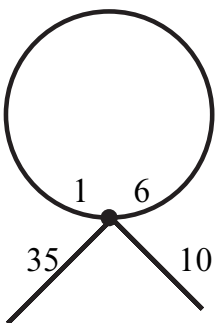

(5)

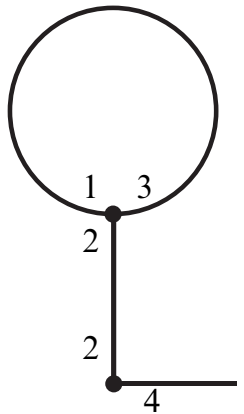

(2)

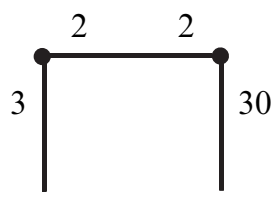

(6)

Figure 8: If $\Gamma$ contains one of these graphs, $\operatorname{Out}(G)$ contains $F_{2}$.

\section{Slid segments are $(2,2)$-segments}

Let $f$ be a slid $(p, q)$-segment with $p, q \geq 2$. Consider the graph of groups $\Theta$ obtained by collapsing $f$. It has a vertex group $H_{v}$ isomorphic to $\left\langle a, b \mid a^{p}=b^{q}\right\rangle$. Let $e$ be an edge of $\Gamma$ that may slide across $f$, viewed as an edge of $\Theta$. Its group $G_{e}$ is generated by a power of $a^{p}$, so is central in $H_{v}$. By Lemma 3.2, the group $\mathcal{T}(\Theta) \subset \operatorname{Out}(G)$ maps onto

$$
J=Z_{H_{v}}\left(G_{e}\right) /\left\langle Z\left(H_{v}\right), Z\left(G_{e}\right)\right\rangle=\left\langle a, b \mid a^{p}=b^{q}=1\right\rangle .
$$

If $(p, q) \neq(2,2)$, the group $J$ contains $F_{2}$, so $\operatorname{Out}(G)$ contains $F_{2}$.

Remark 6.2 For future reference, note that $\mathcal{T}(\Theta)$ contains $\mathcal{T}(\Gamma)$ by Remark 3.1, and furthermore the image of $\mathcal{T}(\Gamma)$ in $J$ is finite. To see this, recall that $\mathcal{T}(\Gamma)$ is generated by the groups $Z_{G_{o\left(f^{\prime}\right)}}\left(G_{f^{\prime}}\right)$ with $f^{\prime} \neq f, \bar{f}$ (Remark 3.1). All these groups have trivial image in $J$, except $Z_{G_{o(e)}}\left(G_{e}\right)$ whose image is finite. 


\section{Slid loops are $(1, q)-$ loops}

We assume that $e$ slides across a $(p, q)$-loop $f$ with $2 \leq p \leq|q|$, and we show that $\operatorname{Out}(G)$ contains $F_{2}$. If $|q| \geq 3$, we create a slid $(p, q)$-segment by performing an expansion move (replacing the loop by a $(p, q)$-segment and a $(1,1)$-segment), and we apply the previous argument (which is valid even if the labelled graph is not reduced). If $f$ is a $(2, \pm 2)$-loop, we collapse it and we apply Lemma 3.2. We now have $H_{v}=\left\langle a, t \mid t a^{2} t^{-1}=a^{ \pm 2}\right\rangle$, and $H_{e}$ is generated by a power of $a^{2}$. The quotient $Z_{H_{v}}\left(G_{e}\right) /\left\langle Z\left(H_{v}\right), Z\left(G_{e}\right)\right\rangle$ is isomorphic to $\mathbb{Z} * \mathbb{Z} / 2 \mathbb{Z}$, so contains $F_{2}$.

\section{Pseudo-ascending loops are $(p, \pm p)$-loops or $(1, q)$-loops}

This amounts to showing that $\Gamma$ cannot contain an $(r, r s)$-loop with $r \geq 2$ and $|s| \geq 2$. If it does, write $G=G_{1} *\langle a\rangle G_{2}$, with $G_{1}=\left\langle a, t \mid t a^{r} t^{-1}=a^{r s}\right\rangle$. By [7], there exist two automorphisms of $G_{1}$ fixing $a$ and generating a free subgroup of rank 2 in $\operatorname{Out}\left(G_{1}\right)$ (in the notation of [7], set $\alpha^{r}=\varphi_{0}=\tau=1$ to see that the subgroup of $\operatorname{Out}\left(G_{1}\right)$ generated by $\alpha$ and $\gamma_{2}$ maps onto $\left.\mathbb{Z} / r \mathbb{Z} * \mathbb{Z} / s^{2} \mathbb{Z}\right)$. Extend the automorphisms by the identity on $G_{2}$ and check that they generate $F_{2} \subset \operatorname{Out}(G)$.

Here is another argument, valid when $r$ or $|s|$ is bigger than 2: perform an expansion and a slide to obtain a graph with a slid $(r, s)$-segment.

\section{Slid edges are disjoint}

We argue by way of contradiction. There are several cases to consider (they are pictured from right to left on the top half of Figure 8). First suppose that $v$ belongs to a slid $(2,2)$-segment $f$ and a $(1, q)$-loop $f^{\prime}$. If $q$ is even, one may slide $f^{\prime}$ across $f$ and then collapse $f^{\prime}$. This creates a $(2,2 q)$-loop, a contradiction. If $q$ is odd, some other edge may slide across $f$. Sliding $f$ around $f^{\prime}$ makes $f$ a slid $(2,2 q)$-segment, a contradiction if $|q|>1$. If $q= \pm 1$, collapse both $f$ and $f^{\prime}$ and apply Lemma 3.2.

Now suppose $v$ belongs to a $(1, q)$-loop $f$ and a $(1, r)$-loop $f^{\prime}$. If $|q| \geq 2$, sliding $f^{\prime}$ around $f$ makes it a $(q, r)$-loop, and then sliding $\bar{f}^{\prime}$ twice makes it a $\left(q, q^{2} r\right)-$ loop. If $|q|=|r|=1$, we may write $G$ as an amalgam $G_{1} *\langle a\rangle G_{2}$, with $G_{2}=\left\langle a, t, t^{\prime}\right| t t^{-1}=$ $\left.a^{ \pm 1}, t^{\prime} a t^{\prime-1}=a^{ \pm 1}\right\rangle$. It is easy to embed $F_{2}$ into $\operatorname{Out}(G)$ by using automorphisms of $G_{2}$ fixing $a$.

Finally, suppose that $(2,2)$-segments $f$ and $f^{\prime}$ have a vertex $v$ in common. We may assume that their other endpoints are distinct, as otherwise sliding $f$ across $f^{\prime}$ would create a slid $(2, \pm 2)$-loop. 
The fundamental group of the subgraph of groups $f \cup f^{\prime}$ is $J=\left\langle a, b, c \mid a^{2}=b^{2}=c^{2}\right\rangle$. Consider the following automorphisms $\alpha, \beta$ of $J: \alpha$ fixes $a$ and $b$ and conjugates $c$ by $b a$, while $\beta$ fixes $b, c$ and conjugates $a$ by $b c$. They extend to automorphisms of $G$ (they are twists in the graph of groups obtained from $\Gamma$ by collapsing $f^{\prime}$ and $f$ respectively). We claim that $\alpha, \beta$ generate a free nonabelian subgroup of $\operatorname{Out}(J)$ (hence also of $\operatorname{Out}(G)$ because $J$ is its own normalizer).

Indeed, consider $\bar{J} \simeq \mathbb{Z} / 2 \mathbb{Z} * \mathbb{Z} / 2 \mathbb{Z} * \mathbb{Z} / 2 \mathbb{Z}$ obtained by adding the relation $a^{2}=1$. Let $J^{+} \subset \bar{J}$ be the subgroup of index 2 consisting of elements of even length. It is free with basis $\{\bar{a} \bar{b}, \bar{b} \bar{c}\}$. With respect to this basis, $\alpha$ acts on the abelianization of $J^{+}$ as the matrix $\left(\begin{array}{ll}1 & 2 \\ 0 & 1\end{array}\right), \beta$ acts as $\left(\begin{array}{ll}1 & 0 \\ 2 & 1\end{array}\right)$, and inner automorphisms of $\bar{J}$ act as \pm Id. It follows that there is no non-trivial relation between $\alpha$ and $\beta$ in $\operatorname{Out}(J)$.

\section{Labels near a $(1, q)$-loop}

Let $v$ be the basepoint of a $(1, q)$-loop. We already know that no other label $r$ at $v$ equals 1 or divides $q$ (it would be carried by a slid edge). Suppose that $r$ divides some $q^{n}$. Let $\ell$ be a prime divisor of $r$. Expand $v$ so as to create a $(1, \ell)-$ segment, and collapse the $\left(1, \frac{q}{\ell}\right)$-edge (see Figure 9). The new labelled graph is isomorphic to $\Gamma$, except that $r$ has been divided by $\ell$ and other indices at $v$ have been multiplied by $\frac{q}{\ell}$. Repeat this operation until $r$ divides $q$, a case already ruled out.

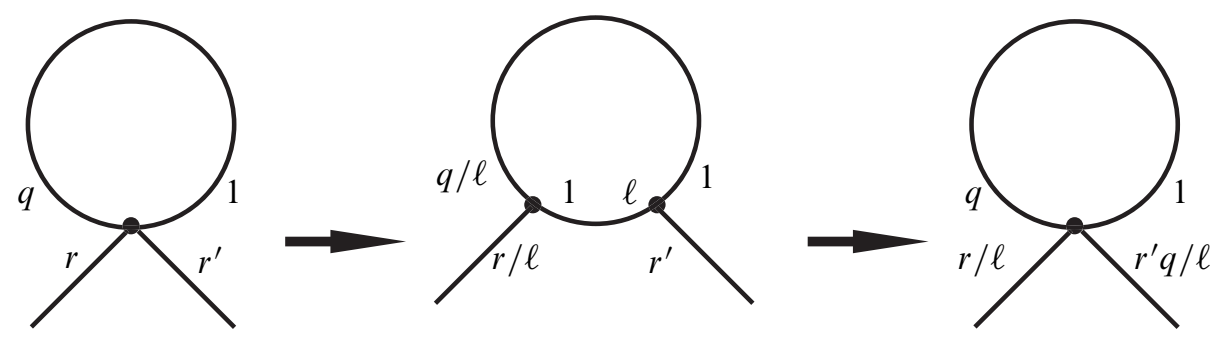

Figure 9: Replacing $r$ by $r / \ell$.

Now let $r, s$ be labels at $v$ carried by edges $e, f$, with $s \mid r q^{n}$. If $f \neq \bar{e}$, we can make $e$ a slid edge by sliding it $n$ times across the loop. If $f=\bar{e}$, we can create an $\left(s, r q^{n+1}\right)$-loop, contradicting (3).

\section{Labels near a $(2,2)$-segment}

Let $v w$ be a $(2,2)$-segment. We already know that all other labels at $v$ and $w$ are bigger than 2 in absolute value. Furthermore, if $r, s$ are labels at the same vertex ( $v$ or $w$ ), and $r \mid s$, then $r= \pm s$ and they are carried by a loop. Assertion (5) of the theorem 
follows: since $s$ is even, one can perform a slide across $v w$ so that $s$ becomes a label at $v$.

\section{Groups with $\operatorname{Out}(G) \not \supset F_{2}$}

In this section, we complete the proof of Theorem 1.6 by showing the following.

Theorem 7.1 If a non-elementary GBS group $G$ is represented by a reduced labelled graph $\Gamma$ satisfying the conclusions of Theorem 6.1, then $\operatorname{Out}(G)$ is virtually nilpotent of class at most 2.

The theorem is true if $G$ is a solvable Baumslag-Solitar group [6], so we rule out this case. As we wish to study the whole automorphism group of $G$, it is important in this proof to think of $\Gamma$ as a marked graph. As usual, we denote by $T$ its Bass-Serre tree.

In general, there are many graphs representing $G$, and the first step in the proof of Theorem 7.1 will be to show that collapsing the slid edges of $\Gamma$ yields a marked graph of groups $\Theta$ and a (non GBS) tree $S$ which are canonical (they do not depend on $\Gamma$ ). In the language of [11], we first show that all possible trees $S$ belong to the same deformation space, and then using [23] that there is only one reduced tree in that space. In particular $\operatorname{Out}(G)=\operatorname{Out}^{S}(G)$, and we shall conclude the proof of Theorem 7.1 by showing that $\operatorname{Out}^{S}(G)$ is virtually nilpotent.

We know that the slid edges of $\Gamma$ are disjoint, and are either $(2,2)$-segments or $(1, q)$-loops. Define $\Theta$ and $S$ by collapsing them.

Consider edge and vertex groups of $\Theta$. Edge groups are cyclic. Non-cyclic vertex groups are Klein bottle groups $\left\langle a, b \mid a^{2}=b^{2}\right\rangle$ arising from collapsed (2,2)-segments, and solvable Baumslag-Solitar groups $B S(1, q)=\left\langle a, t \mid t a t^{-1}=a^{q}\right\rangle$ arising from collapsed $(1, q)$-loops. Note two special cases: $\mathbb{Z}^{2}$ if $q=1$, a Klein bottle group if $q=-1$.

It is useful to think of $B S(1, q)$, for $|q| \geq 2$, as the subgroup of the affine group of $\mathbb{R}$ generated by $a: x \mapsto x+1$ and $t: x \mapsto q x$. It consists of all maps of the form $x \mapsto q^{\alpha} x+\beta$ with $\alpha \in \mathbb{Z}$ and $\beta \in \mathbb{Z}\left[\frac{1}{|q|}\right]$. One deduces, for instance, that powers

$a^{i}, a^{j}$ are conjugate if and only if $\frac{i}{j}$ is a power of $q$. The element $a^{r}$ has an $s$-root if and only if $s$ divides some $r q^{n}$; the root is then unique.

Lemma 7.2 The set of vertex stabilizers of $S$ does not depend on the marked graph $\Gamma$. In particular, it is $\operatorname{Out}(G)$-invariant. 
Proof By Corollary 2.2, it suffices to show that the vertex stabilizers of $S$ are determined by the elliptic subgroups of $T$. This will be done using the tree $T$, but the description of vertex stabilizers will involve only the algebraic structure of the set of elliptic subgroups.

Let $v$ be a vertex of $T$, and $\bar{v}$ its projection in $\Gamma$. We denote by $\pi: T \rightarrow S$ the collapse map. We want to understand the stabilizer $H_{v}$ of $\pi(v)$ in $S$. It is also the stabilizer of the subtree $\pi^{-1}(\pi(v)) \subset T$.

If no strict ascending loop is attached at $\bar{v}$, the stabilizer $G_{v}$ of $v$ is a maximal elliptic subgroup of $T$. Conversely every maximal elliptic subgroup arises in this way. If a $(1, q)$-loop with $|q| \geq 2$ is attached at $\bar{v}$, there is no maximal elliptic subgroup containing $G_{v}$; if $T^{\prime}$ is another GBS tree, then $G_{v}$ is not necessarily a vertex stabilizer of $T^{\prime}$ (see [13]).

We first determine the group $H_{v}$ containing a maximal elliptic subgroup $G_{v}$.

Consider the normalizer $N\left(G_{v}\right)$. If it is $\mathbb{Z}^{2}$ or a Klein bottle group, then $\bar{v}$ bounds a $(1, \pm 1)$-loop and $H_{v}=N\left(G_{v}\right)$. Otherwise, $N\left(G_{v}\right)=G_{v}$. Let $a$ be a generator of $G_{v}$. Then $H_{v}$ is the centralizer $Z\left(a^{2}\right)$ if $\bar{v}$ bounds a slid $(2,2)$-edge, $G_{v}$ if not.

To decide which (in terms of $G_{v}$ only), first observe that $\bar{v}$ bounds a $(2,2)$-edge (slid or not) if and only if $Z\left(a^{2}\right)$ is a Klein bottle group. Assuming it is, consider (as in [16]) the set of groups of the form $Z\left(a^{2}\right) \cap K$, where $K$ is an elliptic subgroup of $T$ not contained in $Z\left(a^{2}\right)$. It is easy to see that the edge is slid if and only if some maximal element of this set (ordered by inclusion) is contained in $\left\langle a^{2}\right\rangle$.

Now suppose that a $(1, q)$-loop with $|q| \geq 2$ is attached at $\bar{v}$. Then $H_{v}=\langle a, t|$ $\left.t a t^{-1}=a^{q}\right\rangle$, where $a$ is a generator of $G_{v}$.

Condition (4) of Theorem 6.1 implies that $\pi(v)$ is the only point of $S$ fixed by $a^{\ell}$ if $\ell$ divides a power of $q$ : stabilizers of edges adjacent to $\pi(v)$ in $S$ are conjugate in $H_{v}$ to $\left\langle a^{r}\right\rangle$, where $r$ does not divide any $q^{n}$; since $a^{\ell}$ is not conjugate (in $H_{v}$ ) to a power of such an $a^{r}$, it cannot fix an edge.

As in [13], say that an elliptic subgroup $K$ of $T$ is vertical if any elliptic subgroup $K^{\prime}$ containing $K$ is contained in a conjugate of $K$. For the action of $H_{v}$ on $T$, the subgroup $\left\langle a^{\ell}\right\rangle$ is vertical if and only if $\ell$ divides a power of $q$. We show that the same result holds for the action of $G$ on $T$.

Suppose that $\ell$ divides a power of $q$. If $K^{\prime} \supset\left\langle a^{\ell}\right\rangle$ is elliptic (in $T$, hence also in $S$ ), then $\pi(v)$ is the only point of $S$ fixed by $K^{\prime}$, so $K^{\prime}$ fixes a point $w \in \pi^{-1}(\pi(v)) \subset T$. The stabilizer of $w$ is conjugate to $\langle a\rangle$ in $H_{v}$, and $\langle a\rangle$ is contained in a conjugate of $\left\langle a^{\ell}\right\rangle$ because $\ell$ is a power of $q$. Thus $K^{\prime}$ is contained in a conjugate of $\left\langle a^{\ell}\right\rangle$, and 
$\left\langle a^{\ell}\right\rangle$ is vertical as required. Conversely, if $\left\langle a^{\ell}\right\rangle$ is vertical, then it contains a conjugate $g\langle a\rangle g^{-1}$ with $g \in G$. Since $\pi(v)$ is the only point of $S$ fixed by $a$, we have $g \in H_{v}$ and we deduce that $\ell$ divides a power of $q$.

We now conclude the proof, by characterizing the vertex stabilizer $H_{v}$ of $S$ containing a vertical subgroup $K \subset G$ which is not maximal elliptic. We know that $K$ is generated by $a^{\ell}$, where $a$ generates a vertex stabilizer $G_{v}$, a $(1, q)$-loop with $|q| \geq 2$ is attached to $\bar{v}$, and $\ell$ divides a power of $q$. In particular, $K$ fixes a unique point $\pi(v) \in S$.

The stabilizer $H_{v}$ of $\pi(v)$ is isomorphic to $B S(1, q)$. The set of elements of $H_{v}$ which are elliptic in $T$ is an abelian subgroup (isomorphic to $\mathbb{Z}\left[\frac{1}{|q|}\right]$ ), so $g \mathrm{Kg}^{-1}$ commutes with $K$ if $g \in H_{v}$. Conversely, if $g K^{-1}$ commutes with $K$, then $g \in H_{v}$ because $\pi(v)$ is the only fixed point of $K$ in $S$. We may now characterize $H_{v}$ (independently of $T$ ) as the set of $g \in G$ such that $g K^{-1}$ commutes with $K$.

Lemma 7.3 The $G$-tree $S$ does not depend on $\Gamma$. In particular, $\operatorname{Out}^{S}(G)=\operatorname{Out}(G)$.

Proof We apply the main result of [23]. Since $S$ is reduced (no inclusion $G_{e} \hookrightarrow H_{v}$ is onto), it suffices to check that the following holds. Let $e$ and $f$ be oriented edges of $S$ with the same origin such that $G_{f} \subset G_{e}$; if $e, f$ do not belong to the same $G$-orbit, then $e, \bar{f}$ are in the same orbit and $G_{e}=G_{f}$.

Let $v, w$ be the origins of $e$ and $f$ in $T$. Let $e_{0}, f_{0}$ be the projections in $\Gamma$, and $r, s$ the corresponding labels. We distinguish several cases.

First assume $\bar{v}=\bar{w}$ in $\Gamma$. If no collapsing takes place at $\bar{v}$, or if $\bar{v}$ bounds a $(1, \pm 1)-$ loop, then $G_{f} \subset G_{e}$ implies that $r$ divides $s$. This is possible only if $e_{0}$ and $f_{0}$ are opposite edges forming a $(p, \pm p)$-loop.

Now suppose that $\bar{v}$ bounds a $(1, q)$-loop with $|q| \geq 2$. Write the corresponding vertex stabilizer $H_{v}$ of $S$ as $\left\langle a, t \mid t a t^{-1}=a^{q}\right\rangle$. Then $\left\langle a^{s}\right\rangle$ is conjugate in $H_{v}$ to a subgroup of $\left\langle a^{r}\right\rangle$, so there exists $n$ such that $\frac{s}{n r}$ is a power of $q$. This contradicts Assertion (5) of Theorem 6.1, so this case cannot occur.

Finally, suppose that $\overline{v w}$ is a slid $(2,2)$-edge. The stabilizer of $\pi(v)$ in $S$ is then $H_{v}=\left\langle a, b \mid a^{2}=b^{2}\right\rangle$. The subgroups $G_{e}$ and $G_{f}$ of $H_{v}$ are generated by conjugates of powers of $a$ or $b$. Distinct powers of $a$ (resp. $b$ ) are not conjugate in $H_{v}$, while $a^{i}$ is conjugate to $b^{j}$ only when $i$ and $j$ are equal and even. In particular, $r$ divides $s$. If $e_{0}$ and $f_{0}$ have the same origin $(\bar{v}$ or $\bar{w})$, we conclude as in the first case. If not, then $s$ must be even and we use Assertion (6) of Theorem 6.1.

We may now study $\operatorname{Out}(G)=\operatorname{Out}^{S}(G)$ using the results of [22] recalled in Section 3. In particular, $\rho$ has a restriction $\rho_{1}: \operatorname{Out}_{1}^{S}(G) \rightarrow \prod_{u \in W} \operatorname{Out}\left(H_{u}\right)$ with $\operatorname{Out}_{1}^{S}(G)$ of 
finite index in $\operatorname{Out}(G)$ and $\operatorname{ker} \rho_{1}=\mathcal{T}(S)$ (we denote by $W$ the vertex set of $\Theta$, and by $H_{u}$ the vertex group of $u \in W$ ).

We first show that $\mathcal{T}(S)$ is virtually abelian. It is generated by centralizers of edge groups in vertex groups $H_{u}$. If $H_{u}$ is $\mathbb{Z}$ or $\mathbb{Z}^{2}$, the centralizer is of course $H_{u}$. If $H_{u}=B S(1, q)$ with $|q| \geq 2$, the centralizer is an infinitely generated abelian group isomorphic to $\mathbb{Z}\left[\frac{1}{|q|}\right]$. If $H_{u}$ is a Klein bottle group, the centralizer is $\mathbb{Z}^{2}$ if $u$ comes from a $(1,-1)$-loop, $\mathbb{Z}$ if $u$ comes from a slid $(2,2)$-segment and the edge group is not central, the whole of $H_{u}$ if the edge group is central. Since a Klein bottle group is virtually abelian, so is $\mathcal{T}(S)$.

Remark 7.4 Relations in the presentation of $\mathcal{T}(S)$ come from centers of edge and vertex groups of $\Theta$. Since these centers are $\{1\}, \mathbb{Z}$, or $\mathbb{Z}^{2}$, the group $\mathcal{T}(S)$ is finitely generated if and only if $\Gamma$ contains no strict ascending loop.

Now fix a vertex $u$ of $\Theta$, and define $P_{u} \subset \operatorname{Out}\left(H_{u}\right)$ by projecting the image of $\rho_{1}$. If $H_{u}$ is $\mathbb{Z}$ or a Klein bottle group, $P_{u}$ is finite because $\operatorname{Out}\left(H_{u}\right)$ is finite. We claim that $P_{u}$ is finite also when $H_{u}$ is $B S(1, q)$ with $|q| \geq 2$.

Write $H_{u}=\left\langle a, t \mid t a t^{-1}=a^{q}\right\rangle$. The vertex $u$ is obtained by collapsing a $(1, q)$-loop $f_{u}$ of $\Gamma$. Denote its basepoint by $v$. Since $G$ is assumed not to be solvable, we may consider an edge group $\left\langle a^{r}\right\rangle$, where $r$ is a label near $v$ not carried by $f_{u}$. Its image by an automorphism $\alpha \in P_{u}$ is also an edge group, so $\alpha\left(\left\langle a^{r}\right\rangle\right)$ is conjugate to $\left\langle a^{s}\right\rangle$ for some label $s$ (possibly equal to $r$ ). But $a^{r}$ has an $s$-th root only if $s$ divides some $r q^{n}$, so $r=s$ by Assertion (5) of Theorem 6.1. By uniqueness of roots, $\alpha$ maps $a$ to a conjugate of $a^{ \pm 1}$. Only finitely many outer automorphisms of $H_{u}$ have this property [6], so $P_{u}$ is indeed finite.

The group $P_{u}$ is infinite only when $u$ comes from collapsing a $(1,1)$-loop $f_{u}$. In this case, $H_{u}=\left\langle a, t \mid t a t^{-1}=a\right\rangle$. As above, $a$ must be mapped to a conjugate of $a^{ \pm 1}$, so $P_{u}$ contains with index at most 2 the group generated by the automorphism $D_{f_{u}}$ fixing $a$ and mapping $t$ to $a t$. We view $D_{f_{u}}$ as an automorphism of $G$ (extend it by the identity). It is a twist relative to $\Gamma$, but not to $\Theta$ (Remark 3.1 does not apply here, as $f_{u}$ is not a segment; in general, none of the groups $\mathcal{T}(S), \mathcal{T}(T)$ contains the other).

This analysis shows that $\mathcal{T}(S)$ and the automorphisms $D_{f_{u}}$ associated to $(1,1)$-loops of $\Gamma$ generate a finite index subgroup of $\operatorname{Out}(G)$. We replace $\mathcal{T}(S)$ by an abelian subgroup $\mathcal{T}_{0}(S)$ of finite index, and we complete the proof of Theorem 7.1 by showing that the subgroup generated by $\mathcal{T}_{0}(S)$ and the automorphisms $D_{f_{u}}$ is virtually nilpotent of class $\leq 2$ : every commutator is central. 
Non-commutativity only comes from the fact that $D_{f_{u}}$ may fail to commute with $D(z)$, when $z \in H_{u}$ and $D(z)$ is a twist of $\Theta$ around an edge $e$ with origin $u$. Write $H_{u}=\left\langle a, t \mid t a t^{-1}=a\right\rangle$. The group $G_{e}$ is generated by a power of $a$.

Recall that $D_{f_{u}}$ fixes $a$ and maps $t$ to at. In particular, $D(z)$ commutes with $D_{f_{u}}$ if $z$ is a power of $a$ (both automorphisms belong to $\mathcal{T}(T)$ ). The interesting case is when $z=t$ (geometrically, $u$ carries a 2-torus $T^{2}, e$ carries an annulus attached to a meridian of $T^{2}, D_{f_{u}}$ is a Dehn twist in $T^{2}$ around a meridian, and $D(t)$ drags the annulus around $T^{2}$ along the longitude). But conjugating $D(t)$ by $D_{f_{u}}$ gives $D(t a)$, so the commutator of $D(t)$ and $D_{f_{u}}$ is $D(a)$, a central element. This easily implies that every commutator is central, completing the proof of Theorem 7.1.

\section{Further results}

\section{Nilpotent vs abelian}

Corollary 8.1 If $G$ is represented by a reduced labelled graph with no $(1,1)-l o o p$, then $\operatorname{Out}(G)$ contains $F_{2}$ or is virtually abelian.

This follows immediately from the proof of Theorem 7.1. More generally, if $\operatorname{Out}(G)$ does not contain $F_{2}$, it is virtually abelian if and only if every commutator $D(a)$ as in the last paragraph of the proof has finite order. This happens in particular when the basepoint of every $(1,1)$-loop has valence 3 . See Remark 3.4 for a more complete discussion.

\section{Finite generation}

Here is a general fact.

Proposition 8.2 Let $\Gamma$ be a labelled graph representing a $G B S$ group $G$. If $\Gamma$ contains a strict ascending loop, but $G$ is not a solvable Baumslag-Solitar group, then $\operatorname{Out}(G)$ has an infinitely generated abelian subgroup.

Proof Collapse the loop and apply Lemma 3.2 to an edge $e$ with origin at the collapsed vertex $v$ (there is such an edge because $G$ is not solvable). We have $H_{v}=B S(1, q)$ with $|q| \geq 2$, and $Z_{H_{v}}\left(G_{e}\right)$ is infinitely generated abelian (it is isomorphic to $\mathbb{Z}\left[\frac{1}{|q|}\right]$ ). The center of $H_{v}$ is trivial, and the center of $G_{e}$ is cyclic. The subgroup of $\mathcal{T}(\Theta)$ generated by $Z_{H_{v}}\left(G_{e}\right)$ is isomorphic to $\mathbb{Z}\left[\frac{1}{|q|}\right]$, or is an infinite abelian torsion group. 
From the proof of Theorem 7.1 we get this Corollary.

Corollary 8.3 Let $\Gamma$ be a reduced labelled graph representing a non-solvable GBS group $G$ with $\operatorname{Out}(G)$ virtually nilpotent. The group $\operatorname{Out}(G)$ is finitely generated if and only if $\Gamma$ contains no strict ascending loop.

Proof We have seen that $\operatorname{Out}(G)$ is generated by the union of $\mathcal{T}(S)$ and a finite set, so by virtual nilpotence $\operatorname{Out}(G)$ is finitely generated if and only if $\mathcal{T}(S)$ is finitely generated. The corollary now follows from Remark 7.4.

Corollary 8.4 If no label of $\Gamma$ equals 1 , then $\operatorname{Out}(G)$ contains $F_{2}$ or is finitely generated and virtually abelian.

In the virtually abelian case, the torsion-free rank may be computed from $k$ and the labels near the $(2,2)$-edges.

\section{Algebraic rigidity}

Theorem 8.5 If the GBS group $G$ is not a solvable Baumslag-Solitar group, the following are equivalent:

(1) $G$ is algebraically rigid (there is only one reduced GBS tree).

(2) The deformation space $P \mathcal{D}$ is a finite complex.

(3) $\operatorname{Out}(G)$ is virtually $\mathbb{Z}^{k}$ (with $k$ defined in Proposition 3.3).

(4) Let $\Gamma$ be any reduced labelled graph representing $G$. If e, $f$ are distinct oriented edges of $\Gamma$ with the same origin $v$, and the label of $f$ divides that of $e$, then either $e=\bar{f}$ is a $(p, \pm p)$-loop with $p \geq 2$, or $v$ has valence 3 and bounds a $(1, \pm 1)-$ loop.

\section{Remarks}

- If $G$ is unimodular, (3) $\Leftrightarrow$ (4) follows from Theorem 4.4 and (Pettet [29, Corollary 5.14]).

- Suppose $|n| \geq 2$. Then $B S(1, n)$ is algebraically rigid if and only if $|n|$ is prime [23], while $\operatorname{Out}(B S(1, n))$ is virtually $\mathbb{Z}^{k}$ (ie finite) if and only if $|n|$ is a prime power [6]. 
Proof The equivalence (1) $\Leftrightarrow$ (4) is in [23], and (1) $\Rightarrow(2) \Rightarrow$ (3) follows from Section 3 and Section 5. We prove (3) $\Rightarrow$ (4).

Suppose that $\operatorname{Out}(G)$ is virtually $\mathbb{Z}^{k}$ (equivalently, $\mathcal{T}(\Gamma)$ has finite index in $\operatorname{Out}(G)$ ). Let $e, f$ be adjacent edges with $\lambda_{f} \mid \lambda_{e}$. By Theorem 6.1 and Proposition 8.2, the edge $f$ must be a slid $(2,2)$-segment or a $(p, \pm p)$-loop.

It cannot be a segment because of Remark 6.2: after collapsing $f$, the group $\mathcal{T}(\Theta) \subset$ $\operatorname{Out}(G)$ would map onto the infinite dihedral group $J=\left\langle a, b \mid a^{2}=b^{2}=1\right\rangle$ with the image of $\mathcal{T}(\Gamma)$ finite, a contradiction. To prove (4), there remains to show that the basepoint of any $(1, \pm)$-loop has valence 3 .

Let $f$ be a $(1, \varepsilon)$-loop, with $\varepsilon= \pm 1$, let $v$ be its basepoint, let $e_{1}, \ldots, e_{n}$ be the oriented edges with origin $v$ (other than $f, \bar{f}$ ). We must show $n=1$.

First consider the subgroup $\mathcal{T}_{0}$ of $\mathcal{T}=\mathcal{T}(\Gamma)$ generated by the twists $D_{e_{i}}$ and the twists around edges with origin other than $v$. The group $\mathcal{T}$ is generated by $\mathcal{T}_{0}$ and the twists $D_{f}, D_{\bar{f}}$. The only relations involving $D_{f}, D_{\bar{f}}$ are $D_{f}+\varepsilon D_{\bar{f}}=0$ (edge relation) and $D_{f}+D_{\bar{f}}+\sum_{i} D_{e_{i}}=0$ (vertex relation). It follows that $\mathcal{T}_{0}$ has index at most 2 in $\mathcal{T}$ if $\varepsilon=-1$, that $\mathcal{T}$ is the direct sum of $\mathcal{T}_{0}$ and the infinite cyclic group generated by $D_{f}$ if $\varepsilon=1$.

Now let $\Theta$ be the graph of groups obtained by collapsing $f$, and consider $\mathcal{T}^{\prime}=\mathcal{T}(\Theta)$. We will see that it is generated by $\mathcal{T}_{0}$ together with extra twists $D_{i}^{\prime}$ around the edges $e_{i}$ (note that edge group centralizers are bigger in $\Theta$ than in $\Gamma$ ). To describe $D_{i}^{\prime}$ precisely, we distinguish two cases.

If $\varepsilon=-1$, the vertex group $H_{v}$ of $v$ in $\Theta$ is a Klein bottle group $\left\langle a, t \mid t a t^{-1}=a^{-1}\right\rangle$. Its center is $\left\langle t^{2}\right\rangle$. The edge groups $G_{e_{i}}$ are generated by powers of $a$. Their centralizer in $H_{v}$ is the free abelian group generated by $a$ and $t^{2}$. In this case, $D_{i}^{\prime}$ is the twist by $t^{2}$ around $e_{i}$. The only relation involving $D_{i}^{\prime}$ is the vertex relation $\sum_{i} D_{i}^{\prime}=0$, so $\mathcal{T}^{\prime}$ is the direct sum of $\mathcal{T}_{0}$ and $\mathbb{Z}^{n-1}$. As $\mathcal{T}_{0}$ has index at most 2 in $\mathcal{T}$, we must have $n=1$ since $\mathcal{T}$ has finite index $\operatorname{in} \operatorname{Out}(G)$.

If $\varepsilon=1$, we still have $\mathcal{T}^{\prime}=\mathcal{T}_{0} \oplus \mathbb{Z}^{n-1}$ (the vertex group $H_{v}$ is $\mathbb{Z}^{2}=\langle a, t\rangle$, and $D_{i}^{\prime}$ is the twist by $t)$. There is a natural homomorphism from $\left\langle\mathcal{T}, \mathcal{T}^{\prime}\right\rangle$ to $\operatorname{Out}\left(H_{v}\right)$, given by the action on $H_{v}$. The kernel contains $\mathcal{T}^{\prime}$, but its intersection with $\mathcal{T}$ is $\mathcal{T}_{0}$ (as $D_{f}$ acts on $H_{v}$ by fixing $a$ and mapping $t$ to $\left.a t\right)$. Since $\mathcal{T}$ has finite index in $\operatorname{Out}(G)$, hence in $\left\langle\mathcal{T}, \mathcal{T}^{\prime}\right\rangle$, we deduce that $\mathcal{T}_{0}=\mathcal{T} \cap \mathcal{T}^{\prime}$ has finite index in $\mathcal{T}^{\prime}$, so $n=1$.

Combining with Proposition 3.3, we obtain this Corollary.

Corollary 8.6 Let $\Gamma$ be a reduced labelled graph representing a group $G$. The group $\operatorname{Out}(G)$ is finite if and only if one of the following holds: 
(1) $\Gamma$ is a tree with no divisibility relation.

(2) $\Gamma$ is a graph with first Betti number 1, there is no divisibility relation, and $G$ has non-trivial modulus.

(3) $\Gamma$ is obtained from a tree with no divisibility relation by attaching one $(k,-k)-$ loop. If $k \geq 2$, no other index at the attaching point is a multiple or a divisor of $k$. If $k=1$, the loop is attached at a terminal vertex.

\section{On the isomorphism problem}

Given a labelled graph $\Gamma$, it is easy to decide algorithmically whether the associated GBS group $G$ is elementary, solvable, unimodular. By Theorem 6.1 and Theorem 7.1, we may decide whether $\operatorname{Out}(G)$ contains $F_{2}$ or is virtually nilpotent.

The isomorphism problem for GBS groups is the problem of deciding whether two (reduced) labelled graphs represent isomorphic groups or not. It is solved for rigid groups (obviously), for groups with no non-trivial integral modulus [13], and for 2-generated groups [21].

Theorem 8.7 The isomorphism problem is solvable for GBS groups such that $\operatorname{Out}(G)$ does not contain a non-abelian free group.

Proof Let $\Gamma$ be a reduced labelled graph representing $G$. We assume that $\operatorname{Out}(G)$ does not contain $F_{2}$, so $\Gamma$ satisfies all six conditions of Theorem 6.1.

We describe three ways of producing new labelled graphs representing $G$ (besides admissible sign changes). The first one changes the graph, the other two only change the labels.

(1) Sliding an edge across a $(2,2)$-segment: it changes the attaching point of an edge (carrying an even label).

(2) If $v$ is the basepoint of a $(1, q)$-loop, one may multiply or divide by $q$ some other label near $v$, by sliding the corresponding edge around the loop.

(3) If $v$ is the basepoint of a $(1, q)$-loop, one may multiply or divide all other labels near $v$ by any number $p$ dividing $q$, by performing an expansion at $v$ followed by a collapse (see Figure 10; this is called an induction move in [23]).

Consider the set $\mathcal{G}$ consisting of all labelled graphs which may be obtained from $\Gamma$ by combining these moves. They are reduced by condition (4) of Theorem 6.1 and it is easy to decide whether a given labelled graph $\Gamma^{\prime}$ belongs to $\mathcal{G}$. We now complete the proof by showing that $\mathcal{G}$ contains all reduced graphs representing $G$. 

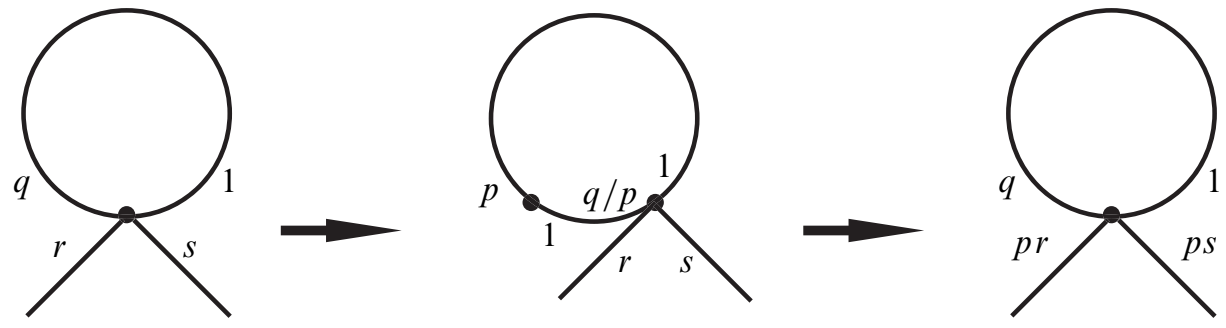

Figure 10: Induction move.

As above, consider the graph of groups $\Theta$ and the Bass-Serre tree $S$ obtained by collapsing the slid edges of $\Gamma$. We have seen that $S$ does not depend on the graph $\Gamma$ used to construct it (Lemma 7.3). It thus suffices to show that the various ways of blowing up $S$ into a GBS-tree differ by the moves mentioned above.

First consider a vertex group $H_{v}$ obtained by collapsing a $(2,2)$-edge. It is a Klein bottle group $\left\langle a, b \mid a^{2}=b^{2}\right\rangle$. The generator of any adjacent edge group is conjugate to some $a^{i}$ or $b^{j}$. Distinct powers of $a$ (resp. $b$ ) are not conjugate in $H_{v}$, while $a^{i}$ is conjugate to $b^{j}$ only when $i$ and $j$ are equal and even. It follows that all ways of blowing up $v$ differ by slides across the $(2,2)$-edge.

Now consider a vertex group $H_{v}=\left\langle a, t \mid t a t^{-1}=a^{q}\right\rangle$ obtained by collapsing a $(1, q)-$ loop of $\Gamma$. Let $T^{\prime}$ be another GBS-tree, associated to a labelled graph $\Gamma^{\prime}$. As in the proof of Lemma 7.2, we cannot say that $a$ generates a vertex group of $T^{\prime}$, only that some $a^{i}$, with $i$ dividing a power of $q$, does.

Suppose for a moment $i=1$. The generator of any edge group adjacent to $v$ is conjugate to a power of $a$. As $a^{m}, a^{n}$ are conjugate in $H_{v}$ only if $\frac{m}{n}$ is a power of $q$, the labelled graphs $\Gamma, \Gamma^{\prime}$ differ by moves of type (2) near the $(1, q)$-loop.

If $i \neq 1$, it is a product of divisors of $q$, and $\Gamma$ and $\Gamma^{\prime}$ differ by moves of types (2) and (3).

Remark The same technique may be used when $G$ is represented by a graph $\Gamma$ satisfying the six conditions of Theorem 6.1, but with arbitrary slid segments allowed in condition (1) (not only $(2,2)$-segments). Condition (6) must then be rephrased as follows: Let $v w$ be a $(p, q)$-edge. Let $r$ be a label at $v$, and $s$ a label at $w$. If $q \mid s$ and $q r \mid s p$, then $q r=s p$ and the labels are carried by the same non-oriented edge. 


\section{Open questions}

\section{Classification}

The classification of GBS groups up to quasi-isometry is known (Farb-Mosher, Whyte). In particular, all non-solvable non-unimodular GBS groups are quasi-isometric [34]. On the other hand, we already mentioned that the isomorphism problem (to decide whether two labelled graphs define isomorphic groups) is solved in special cases but open in general.

A related problem is whether there are only finitely many labelled graphs representing a given $G$. The answer is yes when $G$ is rigid, or has no non-trivial integral modulus [13]. Another example is $G=\left\langle a, b, c \mid a^{2}=b^{14}, b^{2}=c^{2}, t b^{3} t^{-1}=c^{15}\right\rangle$.

Nothing is known about the commensurability problem (to decide whether two labelled graphs define commensurable groups) for non-solvable non-unimodular GBS groups. K Whyte claimed to the author that different Baumslag-Solitar groups are not commensurable (private communication, 2002).

\section{Automorphisms}

When is $\operatorname{Out}(G)$ finitely generated? Clay [3] has a geometric way of showing that $\operatorname{Out}(B S(2,4))$ is not (a result first proved in [7]). Given $n$, does there exist $G$ such that $\operatorname{Out}(G)$ is of type $F_{n}$ but not $F_{n+1}$ ?

When is $\operatorname{Out}(G)$ virtually torsion-free? It is when $G$ is unimodular (Theorem 4.4), it is not when $G=B S(2,4)$. What if there is no non-trivial integral modulus? When $\operatorname{Out}(G)$ is virtually torsion-free, can one compute its virtual cohomological dimension?

A main result of the present paper is that $\operatorname{Out}(G)$ contains $F_{2}$ or is virtually nilpotent. Does the Tits alternative hold in $\operatorname{Out}(G)$ ? $\operatorname{Can} \operatorname{Out}(G)$ contain solvable subgroups which are not virtually nilpotent?

\section{Algebraic properties}

A GBS group $G$ is residually finite if and only if it is unimodular or solvable (this is an unpublished result of D Wise, a proof will be included in [21]). When is $G$ Hopfian?

In [21], we compute the minimum number of elements needed to generate $G$. Can one compute the minimum number of relators? This question is related to the classification of one-relator groups with non-trivial center. Such a group is a GBS group of a special form (Pietrowski [31]), but it is not known which groups of that form are one-relator groups. In particular (see McCool [25]): for which values of $\alpha, \beta, \gamma, \delta, \lambda, \mu$ is $\left\langle a, b, c, d \mid a^{\alpha}=b^{\beta}, b^{\gamma}=c^{\delta}, c^{\lambda}=d^{\mu}\right\rangle$ a one-relator group? 


\section{References}

[1] H Bass, R Kulkarni, Uniform tree lattices, J. Amer. Math. Soc. 3 (1990) 843-902 MR1065928

[2] G Baumslag, D Solitar, Some two-generator one-relator non-Hopfian groups, Bull. Amer. Math. Soc. 68 (1962) 199-201 MR0142635

[3] M Clay, Deformation spaces of $G$-trees and automorphisms of Baumslag-Solitar groups arXiv:math.GR/0702582

[4] M Clay, Contractibility of deformation spaces of $G$-trees, Algebr. Geom. Topol. 5 (2005) 1481-1503 MR2186106

[5] M Clay, A Fixed Point Theorem for Deformation Spaces of $G$-trees, Comment. Math. Helv. (to appear) arXiv:math.GR/0502248

[6] D J Collins, The automorphism towers of some one-relator groups, Proc. London Math. Soc. (3) 36 (1978) 480-493 MR0470091

[7] D J Collins, F Levin, Automorphisms and Hopficity of certain Baumslag-Solitar groups, Arch. Math. (Basel) 40 (1983) 385-400 MR707725

[8] M Culler, K Vogtmann, Moduli of graphs and automorphisms of free groups, Invent. Math. 84 (1986) 91-119 MR830040

[9] A L Fel'shtyn, The Reidemeister number of any automorphism of a Gromov hyperbolic group is infinite, Zap. Nauchn. Sem. S.-Peterburg. Otdel. Mat. Inst. Steklov. (POMI) 279 (2001) 229-240, 250 MR1846083

[10] A Fel'shtyn, DL Goncalves, Twisted conjugacy classes of automorphisms of Baumslag-Solitar groups arXiv:math.GR/0405590

[11] M Forester, Deformation and rigidity of simplicial group actions on trees, Geom. Topol. 6 (2002) 219-267 MR1914569

[12] M Forester, On uniqueness of JSJ decompositions of finitely generated groups, Comment. Math. Helv. 78 (2003) 740-751 MR2016693

[13] M Forester, Splittings of generalized Baumslag-Solitar groups, Geom. Dedicata 121 (2006) 43-59

[14] N D Gilbert, J Howie, V Metaftsis, E Raptis, Tree actions of automorphism groups, J. Group Theory 3 (2000) 213-223 MR1753479

[15] V Guirardel, A very short proof of Forester's rigidity result, Geom. Topol. 7 (2003) 321-328 MR1988289

[16] V Guirardel, G Levitt, Deformation spaces of trees, Geometry, Groups, Dynamics (to appear) arXiv:math.GR/0605545

[17] V Guirardel, G Levitt, The outer space of a free product, Proc. London Math. Soc. (to appear) arXiv:math.GR/0501288 
[18] P H Kropholler, Baumslag-Solitar groups and some other groups of cohomological dimension two, Comment. Math. Helv. 65 (1990) 547-558 MR1078097

[19] P H Kropholler, A note on centrality in 3-manifold groups, Math. Proc. Cambridge Philos. Soc. 107 (1990) 261-266 MR1027778

[20] S Krstić, K Vogtmann, Equivariant outer space and automorphisms of free-by-finite groups, Comment. Math. Helv. 68 (1993) 216-262 MR1214230

[21] G Levitt in preparation

[22] G Levitt, Automorphisms of hyperbolic groups and graphs of groups, Geom. Dedicata 114 (2005) 49-70 MR2174093

[23] G Levitt, Characterizing rigid simplicial actions on trees, from: "Geometric methods in group theory", Contemp. Math. 372, Amer. Math. Soc., Providence, RI (2005) 27-33 MR2139674

[24] G Levitt, M Lustig, Most automorphisms of a hyperbolic group have very simple dynamics, Ann. Sci. École Norm. Sup. (4) 33 (2000) 507-517 MR1832822

[25] J McCool, A class of one-relator groups with centre, Bull. Austral. Math. Soc. 44 (1991) 245-252 MR1126363

[26] D McCullough, A Miller, Symmetric automorphisms of free products, Mem. Amer. Math. Soc. 122 (1996) viii+97 MR1329943

[27] D I Moldavanskiü, On the isomorphisms of Baumslag-Solitar groups, Ukrain. Mat. Zh. 43 (1991) 1684-1686 MR1172309

[28] L Mosher, M Sageev, K Whyte, Quasi-actions on trees. I. Bounded valence, Ann. of Math. (2) 158 (2003) 115-164 MR1998479

[29] M R Pettet, Virtually free groups with finitely many outer automorphisms, Trans. Amer. Math. Soc. 349 (1997) 4565-4587 MR1370649

[30] M R Pettet, The automorphism group of a graph product of groups, Comm. Algebra 27 (1999) 4691-4708 MR1709254

[31] A Pietrowski, The isomorphism problem for one-relator groups with non-trivial centre, Math. Z. 136 (1974) 95-106 MR0349851

[32] E Raptis, D Varsos, On the automorphism group of the fundamental group of a graph of polycyclic groups, Algebra Colloq. 4 (1997) 241-248 MR1681543

[33] A Rhemtulla, D Rolfsen, Local indicability in ordered groups: braids and elementary amenable groups, Proc. Amer. Math. Soc. 130 (2002) 2569-2577 MR1900863

[34] K Whyte, The large scale geometry of the higher Baumslag-Solitar groups, Geom. Funct. Anal. 11 (2001) 1327-1343 MR1878322 
Laboratoire de Mathématiques Nicolas Oresme, UMR 6139, BP 5186

Université de Caen, 14032 Caen Cedex, France

levitt@math.unicaen.fr

Proposed: Martin Bridson

Received: 25th November 2005

Seconded: Walter Neumann and Wolfgang Lueck

Revised: 2 February 2007 